\title{
Dehn surgeries on knots creating essential tori, I
}

\author{
C. McA. Gordon And J. LUeCKE ${ }^{1}$
}

\section{Introduction.}

Throughout this paper, $K$ will be a knot in the 3 -sphere $S^{3}$, which is not a torus knot, and whose exterior $E(K)=S^{3}-\operatorname{Int} \operatorname{nhd}(K)$ contains no non-peripheral incompressible torus (equivalently, $K$ is not a satellite knot). By [T], such a knot is hyperbolic in the sense that $S^{3}-K$ has a complete hyperbolic structure, but we will make no use of this fact.

Let $\gamma$ be a slope on $\partial E(K)$ (i.e., the isotopy class of an essential simple loop), and let $K(\gamma)$ be the closed 3-manifold obtained by $\gamma$-Dehn surgery on $K$. Thus $K(\gamma)=E(K) \cup V_{\gamma}$, where $V_{\gamma}$ is a solid torus, glued to $E(K)$ along their boundaries in such a way that $\gamma$ bounds a disk in $V_{\gamma}$. The main aim of the paper is to examine the situation where $K(\gamma)$ contains an incompressible torus.

To state our results, let $\mu$ be the slope of a meridian of $K$, and let $\Delta(\gamma, \mu)$ be the minimal geometric intersection number of $\gamma$ and $\mu$. (If $\gamma$ is parametrized as $m / n \in \mathbb{Q} \cup\{1 / 0\}$ in the usual way [R], with $n \geq 0$, then $\Delta(\gamma, \mu)=n$.)

Theorem 1.1. If $K(\gamma)$ contains an incompressible torus then $\Delta(\gamma, \mu) \leq 2$.

For strongly invertible knots $K$, this was first proved by Eudave-Muñoz [EM1].

There are many examples as in Theorem 1.1 with $\Delta(\gamma, \mu)=1$, the simplest being with $K$ the figure eight knot and $\gamma= \pm 4$. More generally, the situation for alternating knots $K$ is completely described in [Pat]; here $\Delta(\gamma, \mu)$ is always 1 .

There are also examples with $\Delta(\gamma, \mu)=2$, the simplest being where $K$ is the $(-2,3,7)$ pretzel knot and $\gamma=37 / 2$ [HO]. In fact infinitely many such examples have been given by Eudave-Muñoz [EM2].

\footnotetext{
${ }^{1}$ The authors would like to recognize the partial support of NSF grant DMS 9303229 (first author), as well as the Presidential Young Investigator program, Sloan and O'Donnell foundations (second author).
} 
In the case where $\Delta(\gamma, \mu)=2$ we can say more. Suppose that $K(\gamma)$ contains an incompressible torus $\widehat{T}$, and let $K_{\gamma}$ be the core of $V_{\gamma} \subset K(\gamma)$. We assume that $K_{\gamma}$ intersects $\widehat{T}$ transversely, and that $\widehat{T}$ is chosen (among all incompressible tori in $K(\gamma))$ to minimize $t=\left|\widehat{T} \cap K_{\gamma}\right|$. Then $T=\widehat{T} \cap E(K)$ is a properly embedded punctured torus in $E(K)$ with $|\partial T|=t$. Note that $t \geq 1$ by our hypothesis on $K$, and that $t$ is even if $\Delta(\gamma, \mu) \geq 2$. In this paper together with its sequel [GLu1] we shall prove:

Theorem 1.2. Suppose that $K(\gamma)$ contains an incompressible torus, where $\Delta(\gamma, \mu)=2$. Then $t=2$, and $T$ separates $E(K)$ into two genus 2 handlebodies. In particular, $K$ is strongly invertible. Furthermore, the tunnel number of $K$ is at most 2.

Again, it was shown in [EM1] that assuming $K$ to be strongly invertible, $\Delta(\gamma, \mu)=2$ implies $t=2$.

We also consider the situation where $K(\gamma)$ contains an embedded Klein bottle.

Theorem 1.3. If $K(\gamma)$ contains a Klein bottle then $\Delta(\gamma, \mu)=1$.

There are many examples where this occurs, again the simplest being the figure eight knot with $\gamma= \pm 4$.

Corollary 1.4 (Boyer-Zhang [BZ1]). If $K(\gamma)$ is a Seifert fiber space over the 2-sphere with three exceptional fibers of orders $2,2, n$, then $\Delta(\gamma, \mu)=1$.

Examples where $K(\gamma)$ is such a Seifert fiber space are given in $[\mathrm{BH}]$ and [BZ1].

We prove Theorem 1.1 by first showing (Theorem 4.1) that if $\Delta(\gamma, \mu) \geq 3$ then $t=2$, and then showing (Theorem 8.1) that this implies that $K$ is strongly invertible. Theorem 1.1 now follows from [EM1].

Turning to Theorem 1.2, in the present paper we prove the following weak version.

Theorem 1.2'. Suppose that $K(\gamma)$ contains an incompressible torus, where $\Delta(\gamma, \mu)=2$. Then $t=2$ or 4 .

The case $t=4$ will be ruled out in [GLu1]. As the argument in this case is quite special and rather lengthy, we felt that it would make for greater overall clarity if it were treated separately. Thus $t$ must be 2 and the rest of Theorem 1.2 now follows from Theorem 8.1. 
To put our results in a more general context, recall that Thurston has shown that if $K$ is a hyperbolic knot then $K(\gamma)$ is hyperbolic for all but finitely many slopes $\gamma$ [T]. On the other hand, if $K(\gamma)$ fails to be hyperbolic then it either (1) is reducible; or (2) is an atoroidal Seifert fiber space; or (3) contains an incompressible torus; or (4) is a counterexample to the Geometrization Conjecture [T].

It is expected that (1) never occurs; this would follow from the (still unsettled) Cabling Conjecture [GS].

Regarding (2), some results are known: for example $K(\gamma)$ is never $S^{3}$ (for $\gamma \neq \mu)$ [GLu2], or $S^{1} \times S^{2}$ [Ga]. Infinitely many examples exist where $K(\gamma)$ is a lens space [Be], but here the Cyclic Surgery Theorem [CGLS] implies that if $K(\gamma)$ and $K\left(\gamma^{\prime}\right)$ are lens spaces (or, possibly more generally, have finite cyclic fundamental group), then $\Delta(\gamma, \mu)=\Delta\left(\gamma^{\prime}, \mu\right)=\Delta\left(\gamma, \gamma^{\prime}\right)=1$. Restrictions on when $\pi_{1}(K(\gamma))$ can be finite are obtained in [BZ1].

The results of the present paper give some information about case (3). In the special case where $K(\gamma)$ contains an incompressible torus and is also Seifert fibered [BZ2] shows that $\Delta(\gamma, \mu) \leq 1$. In fact, [BZ2] shows that if $K(\lambda)$ is any cyclic surgery then $\Delta(\gamma, \lambda) \leq 1$.

The paper is organized as follows. In Section 2 we describe how the assumption that $K(\gamma)$ contains an incompressible torus leads to the existence of two labelled graphs $G_{Q}$ and $G_{T}$ in the 2-sphere and torus respectively. Then, assuming that $\Delta(\gamma, \mu) \geq 2$, we show how the technology of [GLu4] leads to the existence of a particular kind of configuration in $G_{Q}$ that we call a great web. In Section 3 we show that on the other hand $G_{Q}$ cannot contain certain combinations of Scharlemann cycles [CGLS], nor a configuration that we call an extended Scharlemann cycle. In Section 4 we show how the existence of a great web in $G_{Q}$ together with the results of Section 3 , quickly leads to the conclusion that if $\Delta(\gamma, \mu) \geq 3$ then $t=2$ (Theorem 4.1). Similarly, in Section 5 we prove Theorem $1.2^{\prime}$, that if $\Delta(\gamma, \mu)=2$ then $t=2$ or 4 . In Sections 6 and 7 we consider the situation where $K(\gamma)$ contains a Klein bottle, and prove Theorem 1.3, that we must have $\Delta(\gamma, \mu)=1$. Here, we choose a Klein bottle $\widehat{S}$ in $K(\gamma)$ which intersects $K_{\gamma}$ transversely and minimizes $\left|\widehat{S} \cap K_{\gamma}\right|$. The boundary of a regular neighborhood of $\widehat{S}$ is a torus $\widehat{T}$, meeting $K_{\gamma}$ in $t=2\left|\widehat{S} \cap K_{\gamma}\right|$ points. Again this gives rise to graphs $G_{Q}$, $G_{T}$ as in Section 2, and in Section 6 we examine how the combinatorics of Section 2 and some of the results of Sections 3 and 4 carry over to this case, where $\widehat{T}$ is not necessarily incompressible in $K(\gamma)$. We emphasize that, although it is more natural to first describe the case of an incompressible torus, and then discuss the modifications that are needed in the case of a 
Klein bottle, the results of Sections 6 and 7 are logically independent of those of Section 3, and this allows us to use Theorem 1.3 in that section. In Section 6 we prove Theorem 1.3 when $t \geq 4$, and in Section 7 we deal with the case $t=2$. Finally, in Section 8, we prove Theorem 8.1, and deduce Theorem 1.1 from Theorem 4.1, Theorem 8.1, and the main result of [EM1].

\section{Webs.}

As in Section 1, let $K$ be a non-torus knot whose exterior $E=E(K)$ contains no non-peripheral incompressible torus, and let $\widehat{T}$ be an incompressible torus in $K(\gamma)$ with $t=\left|\widehat{T} \cap K_{\gamma}\right|$ minimal. Then $T=\widehat{T} \cap E$ is an incompressible punctured torus in $E$ with $|\partial T|=t$, each component of $\partial T$ having slope $\gamma$ on $\partial E$. Assume that $\Delta=\Delta(\gamma, \mu) \geq 2$.

Let $p_{+}, p_{-}$be two points in $S^{3}$. We can write $S^{3}-\left\{p_{+}, p_{-}\right\}=\widehat{Q} \times(-1,1)$, where $\widehat{Q}$ is a 2-sphere. Lemma 4.4 of [Ga, p.491] says that we can find a $\widehat{Q}=\widehat{Q} \times\{i\}$ for some $i$ such that:

(1) $\widehat{Q}$ intersects $K$ transversely. Thus $Q=\widehat{Q} \cap E$ is a properly embedded planar surface in $E$ such that each component of $\partial Q$ is a copy of the meridian $\mu$ of $K$.

(2) $Q$ intersects $T$ transversely and no arc component of $Q \cap T$ is parallel in $Q$ to $\partial Q$ or parallel in $T$ to $\partial T$.

Let $G_{Q}$ be the graph in $\widehat{Q}$ obtained by taking as the (fat) vertices the disks $\widehat{Q}-$ Int $Q$ and as edges the arc components of $Q \cap T$ in $\widehat{Q}$. Similarly, $G_{T}$ is the graph in $\widehat{T}$ whose vertices are the disks $\widehat{T}-\operatorname{Int} T$ and whose edges are the arc components of $Q \cap T$ in $\widehat{T}$. We number the components of $\partial Q$ $1,2, \ldots, q$ in the order in which they appear on $\partial E$. Similarly, we number $1,2, \ldots, t$ the components of $\partial T$. This gives a numbering of the vertices of $G_{Q}$ and $G_{T}$. Furthermore, it induces a labelling of the endpoints of edges in $G_{Q}$ and $G_{T}$. For example, an endpoint of an edge in $G_{Q}$ at vertex $x$ will be labelled $y$ if the endpoint represents the intersection of component $x$ of $\partial Q$ with component $y$ of $\partial T$. On a vertex of $G_{Q}\left(G_{T}\right)$ one sees the labels 1 through $t$ ( 1 through $q$, resp.) appearing in order around the vertex, each label appearing $\Delta$ times. See Figure 2.1. Two vertices on $G_{Q}$ are parallel if the ordering of the labels on each is clockwise or the ordering on each is anticlockwise, otherwise the vertices are called antiparallel. The same applies to vertices of $G_{T}$. The graphs $G_{Q}$ and $G_{T}$ then satisfy the following parity rule [GLu2, p.386]: An edge connects parallel vertices on one graph if and only if it connects antiparallel vertices on the other. 


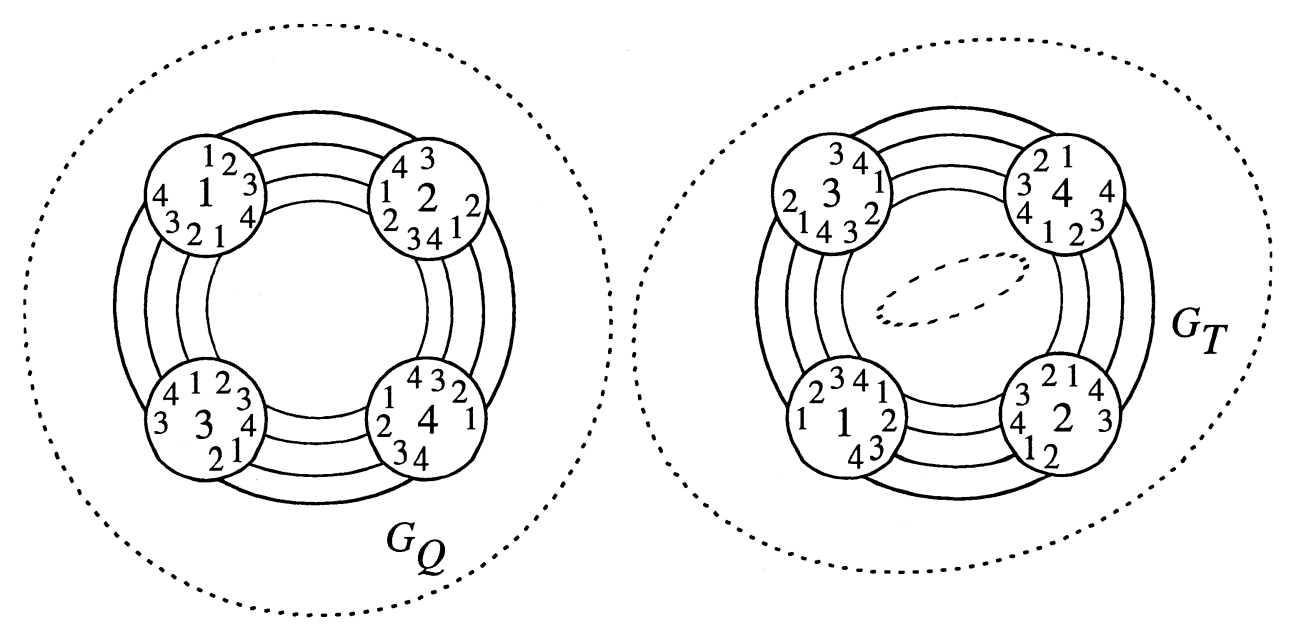

Figure 2.1.

In the remainder of the paper we will write $G$ when we mean to indicate either $G_{Q}$ or $G_{T}$.

The length of a face of $G$ is the number of edges in its boundary (counted with multiplicity). By the choice of $Q$ we see that neither $G_{Q}$ or $G_{T}$ contains a face of length 1 .

Let $x$ be a label of $G$. An $x$-edge in $G$ is an edge with label $x$ at one endpoint. An $x$-cycle is a cycle $\Sigma$ of $x$-edges of $G$ such that all the vertices of $G$ in $\Sigma$ are parallel and which can be oriented so that the tail of each edge has label $x$. A Scharlemann cycle is an $x$-cycle that bounds a disk face of $G$. Each edge of a Scharlemann cycle has the same pair of (adjacent) labels $x, y$ so we refer to such a Scharlemann cycle as an $(x, y)$-Scharlemann cycle (and will often shorten this to $x y$-Scharlemann cycle). The number of edges in a Scharlemann cycle, $\sigma$, is called the length of $\sigma$.

A web, $\Lambda$, in $G_{Q}$ is a connected subgraph of $G_{Q}$ whose vertices are all parallel and such that there are at most $t$ points where the fat vertices of $\Lambda$ are incident to edges of $G_{Q}$ that are not edges of $\Lambda$. We refer to such an edge as a ghost edge of $\Lambda$ and an incidence of a ghost edge with a vertex of $\Lambda$ as a ghost label of $\Lambda$. If $U$ is a component of $Q-\operatorname{nhd}(\Lambda)$ we will refer to $D=\widehat{Q}-U$ as a disk bounded by $\Lambda$.

A great web in $G_{Q}$ is a web with the property that there is a disk bounded by $\Lambda, D_{\Lambda}$, such that $\Lambda$ contains all the edges of $G_{Q}$ that lie in $D_{\Lambda}$.

We assume familiarity with the terminology of [GLu2, Chapter 2]. Because $\Delta=1$ in [GLu2], for this paper one must take the appropriate gen- 
eralizations to the case $\Delta \geq 2$ (this is done in [GLu4] from which in fact many arguments will be taken). To alleviate the ambiguities in this process we make the following comments.

1. The labels of $G_{Q}$ are $1,2, \ldots, t$ and the labels of $G_{T}$ are $1,2, \ldots, q$. If $L$ is a subset of the set of labels of $G$ then each $L$-interval appears $\Delta$ times. Thus an $L$-type is an $|L|$-type and in a star $\mathcal{T}$ representing the $L$-type $\tau$, the dual orientation dictated by $\tau$ and $V(\mathcal{T})$ is assigned to each $L$-interval around $V(\mathcal{T})$.

2. If $\mathcal{T}$ is a star representing some $L$-type then $C(\mathcal{T})$ and $\mathrm{A}(\mathcal{T})$ denote the appropriate subsets of the labels (i.e., each element of $C(\mathcal{T})$ or $A(\mathcal{T})$ appears $\Delta$ times around $V(\mathcal{T})$ ).

Let $\mathbf{q}$ be the set of labels $\{1,2, \ldots, q\}$ of $G_{T}$.

Theorem 2.1. $G_{T}$ does not represent all $\mathbf{q}$-types.

Proof. $\widehat{Q}$ divides $S^{3}$ into black and white sides. In particular $\widehat{Q}$ divides a regular neighborhood of $K$ into 1-handles $H_{i}$ running from component $i$ to $i+1$ of $\partial Q$. The $H_{i}$ are then either black handles or white handles. The qintervals correspond to these $H_{i}$ and are also divided into black q- intervals, $\mathbf{B}$, and the white $\mathbf{q}$-intervals, $\mathbf{W}$. A $\mathbf{B}$-type ( $\mathbf{W}$-type) is a |B|-type [GLu2, p.387] where each coordinate is formally associated with a different black (white) $\mathbf{q}$-interval. In the same way that $\mathbf{B} \cup \mathbf{W}=\{\mathbf{q}$-intervals $\}$, a $\mathbf{B}$-type and a $\mathbf{W}$-type together form a q-type. The faces of $G_{T}$ are divided into black and white faces according to whether the corners of these faces are black or white q-intervals. It is not hard to see then that if there is a collection of faces of $G_{T}$ representing all types then there is either a collection of black faces representing all B-types or a collection of white faces representing all W-types (see Lemma 3.1 of [GLu2] with $N_{0}=\emptyset$ ).

We assume there is a collection $\mathcal{D}$ of black faces of $G_{T}$ representing all B-types. After possibly surgering and replacing $\mathcal{D}$ with a subcollection we may assume that no face of $\mathcal{D}$ intersects $\widehat{Q}$ in its interior (see the proof of part A of Proposition 3.2 of [GLu2]). Let $B$ be the 3-ball on the white side of $\widehat{Q}$ and let $\mathcal{H}$ be the collection of black one-handles $H_{i}$. Let $N$ be a regular neighborhood of $B \cup \mathcal{H} \cup \mathcal{D}$. Then $H_{1}(N)$ is presented by $\mathbb{Z}^{q}$ with relations given by the elements of $\mathcal{D}$.

We now recall

Theorem 2.2. If $\mathcal{A} \subset \mathbb{Z}^{q}$ represents all $\mathbf{q}-$ types then there is a subset $\mathcal{A}_{0}$ of $\mathcal{A}$ such that $\mathbb{Z}^{q}$ modulo the subgroup generated by $\mathcal{A}_{0}$ has non-trivial torsion. 
Proof. This is the main result of [Par].

The above theorem says that there is a submanifold $N^{\prime}$ of $N$ such that $H_{1}\left(N^{\prime}\right)$ has non-trivial torsion. But $N^{\prime}$ is a submanifold of $S^{3}$ and a MayerVietoris calculation shows that this is impossible.

(Theorem 2.1)

Theorem 2.3. Let $L$ be a subset of q. Let $\tau$ be a non-trivial $L$-type. Let $\mathcal{T}$ be a star with $L(\mathcal{T})=L$ and $[\mathcal{T}]=\tau$. Suppose further that

(1) the elements of $C(\mathcal{T})$ correspond to parallel vertices of $G_{Q}$;

(2) the elements of $A(\mathcal{T})$ correspond to parallel vertices of $G_{Q}$;

(3) $G_{T}(L)$ does not represent $\tau$.

Then there is a web $\Lambda$ in $G_{Q}$ such that the set of vertices of $\Lambda$ is a subset of either $C(\mathcal{T})$ or $A(\mathcal{T})$.

Proof. Note: We will blur the distinction here between $C(\mathcal{T})$ and $A(\mathcal{T})$ as labels on $G_{T}$ and as vertices in $G_{Q}$.

We assume $G_{T}$ does not represent $\tau$. Let $s$ be the number of edges of $\Gamma(\mathcal{T})$ whose endpoints are both in $C(\mathcal{T})$ or both in $A(\mathcal{T})$; we refer to these as switch edges. Let $S$ be the number of switches around $\mathcal{T}$, that is $S=\Delta(|C(\mathcal{T})|+|A(\mathcal{T})|)=2 \Delta|C(\mathcal{T})|$. Note that $C(\mathcal{T})$ and $A(\mathcal{T})$ are non-empty since $\tau$ is non-trivial.

Let $i=S / 2-1$. The proof of Lemma 2.3.2 of [GLu2] shows that $s \geq i t$. Thus there are at least $i t / 2$ edges of $G_{T}$ whose labels are in, say, $C(\mathcal{T})$. Set $\Lambda$ to be the subgraph of $G_{Q}$ consisting of these edges (thought of now as edges in $G_{Q}$ ) and the vertices of $C(\mathcal{T})$. There are $|C(\mathcal{T})| \Delta t$ points where edges of $G_{Q}$ are incident to the vertices of $\Lambda$. But at least $2 i t / 2=i t=$ $(S / 2-1) t=|C(\mathcal{T})| \Delta t-t$ of these points come from edges of $\Lambda$. Thus there are at most $t$ points where an edge of $G_{Q}$ not in $\Lambda$ is incident to a vertex of $\Lambda$. After replacing $\Lambda$ by one of its connected components, $\Lambda$ is the desired web.

The proof of Theorem 2.5 will use the following lemma.

Lemma 2.4. Let $\mathcal{R}$ be a star and $L_{0} \subset L(\mathcal{R})$ be a set of labels such that

(1) all the elements of $A(\mathcal{R})-L_{0}$ have the same parity, and 
(2) the exceptional labels of $G\left(L_{0}\right)$ have the same parity and are contained in $C(\mathcal{R})$.

Then there is an $L_{0}$-type $\tau_{0}$ such that any face of $G\left(L_{0}\right)$ representing $\tau_{0}$ contains a face of $G(L(\mathcal{R}))$ representing $[\mathcal{R}]$.

Proof. This is proved on pages 407-408 of [GLu2] beginning just after the proof of Claim 1 on page 407 and ending with the second sentence on page 408 .

Theorem 2.5. Assume $\Delta \geq 2$. Let $D$ be a disk in $\widehat{Q}$ that is either the complement of a small open disk disjoint from $G_{Q}$ or a disk bounded by a web, $\Lambda$. Let $L$ be the set of vertices of $G_{Q}-\Lambda$ in $D$. Then either $G_{Q}$ contains a great web or $G_{T}(L)$ represents all L-types.

Proof. The proof is by induction on $|L|$.

Let $\Lambda$ be the web in the hypothesis of Theorem 2.5 - in the first case of this hypothesis take $\Lambda=\emptyset$. Let $\tau$ be an $L$-type. We want to show there is a face of $G_{T}(L)$ representing $\tau$. There are two cases.

(1) $\tau$ is trivial

(2) $\tau$ is non-trivial

Case (1). $\tau$ is trivial.

A disk face of $G_{T}(L)$ represents the trivial type if and only if all the vertices of $G_{T}$ to which it is incident are parallel. Let $J \subset L$ be the set of vertices of opposite sign to those of $\Lambda$ (if $\Lambda=\emptyset$ let $J$ be the set of all vertices of $G_{Q}$ of some sign). If $J=\emptyset$ then a component of $\Lambda$ is a great web and we are done. So we assume $|J| \geq 1$. Let $\widehat{\Lambda}$ be a component of the subgraph of $G_{Q}$ consisting of vertices $J$ and all the edges of $G_{Q}$ with endpoints on these vertices. We distinguish two subcases, (a) and (b).

(a) Suppose $\widehat{\Lambda}$ is a web. Let $\widehat{D}$ be the disk bounded by $\widehat{\Lambda}$ that is contained in $D$. Then by induction either $G_{Q}$ contains a great web or $G_{T}$ represents all $H$-types where $H \subset L$ is the set of vertices of $G_{Q}-\widehat{\Lambda}$ in $\widehat{D}$. We may assume the latter and conclude there is a face of $G_{T}(H)$ representing the trivial $H$-type. But now filling in this face of $G_{T}(H)$ with the edges of $G_{T}(L)$ we will see a face of $G_{T}(L)$ representing the trivial $L$-type. 
(b) Suppose $\widehat{\Lambda}$ is not a web. Then there are more than $t$ edges of $G_{Q}$ connecting a vertex in $\widehat{\Lambda}$ with an antiparallel vertex in $D$. Let $\Sigma$ be the subgraph of $G_{T}$ consisting of the vertices of $G_{T}$ along with these edges (thought of now as edges in $G_{T}$ ). An Euler characteristic argument says that $\Sigma$ must have a disk face. As the edges of $\Sigma$ connect only parallel vertices, this face of $\Sigma$ (when we add the edges of $G_{T}(L)$ ) will contain a face of $G_{T}(L)$ representing the trivial $L$-type.

Cases (a) and (b) exhaust the possibilities when $\tau$ is trivial.

\section{Case (2). $\tau$ is non-trivial.}

Follow the proof on page 406 of [GLu2], case (2), to construct a sequence of stars $\mathcal{T}_{1}, \ldots, \mathcal{T}_{n}, n \geq 1$, such that

(1) $\left[\mathcal{T}_{1}\right]=\tau,\left[\mathcal{T}_{i}\right]$ is non-trivial, $1 \leq i \leq n$

(2) $\mathcal{T}_{i}=d_{i} \mathcal{T}_{i-1}$ where $d_{i}=d^{ \pm}, 2 \leq i \leq n$

(3) all elements of $C\left(\mathcal{T}_{n}\right)$ have the same parity

(4) all elements of $A\left(\mathcal{T}_{n}\right)$ have the same parity

Set $L_{i}=L\left(\mathcal{T}_{i}\right)$. Assume $f$ is a face of $G_{T}\left(L_{n}\right)$ representing $\mathcal{T}_{n}$. Then $(n-1)$ applications of Corollary 2.4.2 of [GLu2] show that within $f$ we will find a face $f^{\prime}$ of $G_{T}\left(L_{1}\right)=G_{T}(L)$ representing $\tau$ (that $f^{\prime}$ is contained in $f$ is stated in Lemma 2.4.1 of [GLu2]). Thus if we have a face of $G_{T}\left(L_{n}\right)$ representing $\left[\mathcal{T}_{n}\right]$ then we are done. So we assume there are no faces of $G_{T}\left(L_{n}\right)$ representing $\left[\mathcal{T}_{n}\right]$.

By Theorem 2.3, $G_{Q}$ contains a web, $\Omega$, whose vertices are contained in $C\left(\mathcal{T}_{n}\right)$ or $A\left(\mathcal{T}_{n}\right)$. We may assume the vertices of $\Omega$ are contained in $C\left(\mathcal{T}_{n}\right)$ by replacing (if necessary) $\mathcal{T}_{n}$ by $\overline{\mathcal{T}}_{n}$. If $n>1$, this is done by replacing $d_{n}$ $\left(=d^{+}\right)$by $d^{-}$.

Now $\Omega$ is in $D$ and disjoint from $\Lambda$. Let $D_{\Omega}$ be the disk in $D$ bounded by $\Omega$. Let $L_{0}$ be the set of vertices of $G_{Q}-\Omega$ in $D_{\Omega}$. We may assume $L_{0} \neq \emptyset$, otherwise $\Omega$ is a great web.

Let $\mathcal{R}_{1}, \ldots, \mathcal{R}_{n}$ be the sequence of stars corresponding to $\mathcal{T}_{1}, \ldots, \mathcal{T}_{n}$ obtained by taking derivatives relative to $L_{0}$. That is $\mathcal{R}_{1}=\mathcal{T}_{1}, \mathcal{R}_{i}=$ $\left(d_{i}\right)_{L_{0}} \mathcal{R}_{i-1}, 2 \leq i \leq n$. Applying Lemma 2.4 to $L_{0}$ and $\mathcal{R}_{n}$ (to see that conditions (1) and (2) of Lemma 2.4 are satisfied appeal to Proposition 2.1.2 of [GLu2]), we find an $L_{0}$-type $\tau_{0}$ such that any disk face of $G_{T}\left(L_{0}\right)$ representing $\tau_{0}$ contains within it a disk face of $G_{T}\left(L\left(\mathcal{R}_{n}\right)\right)$ representing $\left[\mathcal{R}_{n}\right]$. Since $\left|L_{0}\right|<|L|$, we may now apply the inductive hypothesis to $L_{0}, \tau_{0}$ to conclude 
that either $G_{Q}$ contains a great web or $G_{T}\left(L_{0}\right)$ represents $\tau_{0}$. We assume the latter. By the choice of $\tau_{0}$, this means there is a face of $G_{T}\left(L\left(\mathcal{R}_{n}\right)\right)$ representing $\left[\mathcal{R}_{n}\right]$. We will be done once we prove:

Lemma 2.6. $A$ face of $G_{T}\left(L\left(\mathcal{R}_{n}\right)\right)$ representing $\left[\mathcal{R}_{n}\right]$ contains within it a face of $G_{T}(L)$ representing $\tau$.

Proof. We have

\{exceptional labels of $\left.G_{T}\left(L_{0}\right)\right\} \subset C\left(\mathcal{T}_{n}\right) \subset C\left(\mathcal{T}_{i}\right) \subset C\left(\mathcal{R}_{i}\right)$

(Proposition 2.1.2 of [GLu2]). Thus Lemma 2.2.2 of [GLu2] implies that $\Gamma\left(\mathcal{R}_{i}\right)=\Gamma\left(\left(d_{i}\right)_{L_{0}} \mathcal{R}_{i-1}\right)=\delta_{G_{T}\left(L_{0}\right)} \Gamma\left(\mathcal{R}_{i-1}\right)$ for $2 \leq i \leq n$. Repeated applications of Lemma 2.4.1 of [GLu2] show that each face $E$ of $G_{T}\left(L\left(\mathcal{R}_{n}\right)\right)$ which represents $\left[\mathcal{R}_{n}\right]$ contains within it a face of $G_{T}(L)=G_{T}\left(\mathcal{R}_{1}\right)$ representing $\left[\mathcal{R}_{1}\right]=\tau\left(E\right.$ corresponds to a sink or source of $\left.\Gamma\left(\mathcal{R}_{n}\right)^{*}\right)$.

This completes the proof of Theorem 2.5 .

Corollary 2.7. $G_{Q}$ contains a great web, $\Lambda$.

Proof. This follows from Theorems 2.1 and 2.5.

Note that, since $\Delta \geq 2$ and $G_{Q}$ contains no face of length 1, a great web in $G_{Q}$ must have more than one vertex.

\section{Scharlemann cycles and extended Scharlemann cycles.}

Let $\sigma$ be a Scharlemann cycle in $G_{Q}$. Suppose that $\sigma$ is immediately surrounded by a cycle $\kappa$ in $G_{Q}$, that is, each edge of $\kappa$ is immediately parallel to an edge of $\sigma$ (see Figure 3.1). Then $\kappa$ will be referred to as an extended Scharlemann cycle. This is a slight generalization of the same notion defined in [Wu]. Note that if we orient $\kappa$, then for some labels $x$ and $y$, each edge of $\kappa$ has label $x$ at its tail and label $y$ at its head.

In subsequent sections, the existence of a great web $\Lambda$ in $G_{Q}$ will lead either to the existence of several Scharlemann cycles on distinct pairs of labels or to an extended Scharlemann cycle. These, in turn, will lead to the desired conclusions (usually a contradiction). In this section we explore the consequences of having Scharlemann cycles or extended Scharlemann cycles in $G_{Q}$. 


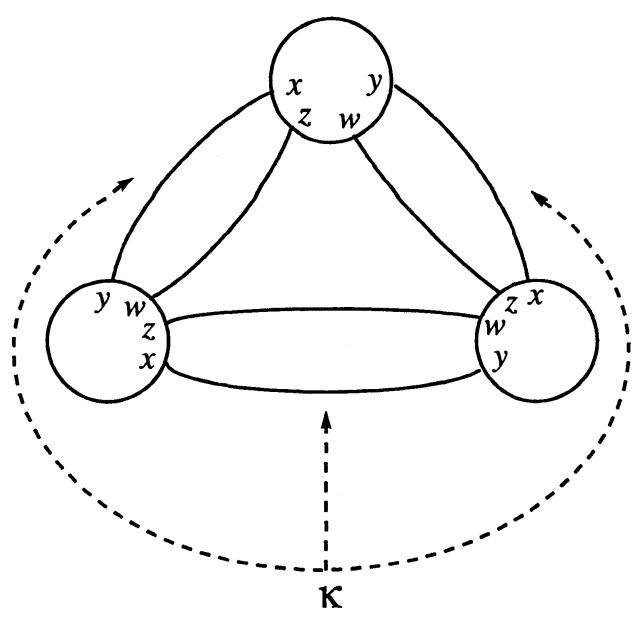

Figure 3.1.

Since $\Delta \geq 2, \widehat{T}$ separates $K(\gamma)$ into two components $X, X^{\prime}$. By the minimality of $\widehat{T}$, we may further assume that every disk face of $G_{Q}$ intersects $\widehat{T}$ in its boundary (i.e., contains no simple closed curves of intersection). Thus every disk face of $G_{Q}$ lies in either $X$ or $X^{\prime}$.

For the remainder of this section, let $H_{i, i+1}$ be that part of $V_{\gamma}$ between consecutive components $i$ and $i+1$ of $\partial T$.

In the next lemma, and in similar contexts throughout the paper, when we talk about a set of edges of $G_{Q}$ as they lie on $\widehat{T}$, it is to be understood that we also include the fat vertices of $G_{T}$ that occur as the endpoints of the edges.

Lemma 3.1. The edges of a Scharlemann cycle in $G_{Q}$ cannot lie in a disk in $\widehat{T}$.

Proof. Without loss of generality assume that $\sigma$ is a Scharlemann cycle in $G_{Q}$ with labels $\{1,2\}$. Let $f$ be the face of $G_{Q}$ bounded by $\sigma$. Assume for contradiction that the edges of $\sigma$ lie in a disk $D$ in $\widehat{T}$. Then $\operatorname{nhd}\left(D \cup H_{12} \cup f\right)$ is a lens space minus an open 3-ball. See Figure 3.2.

The incompressibility of $\widehat{T}$ means that $K(\gamma)$ must be reducible. But this contradicts [GLu3] since $\Delta(\gamma, \mu) \geq 2$.

Theorem 3.2. If $t \geq 4$ then $G_{Q}$ does not contain an extended Scharlemann cycle. 

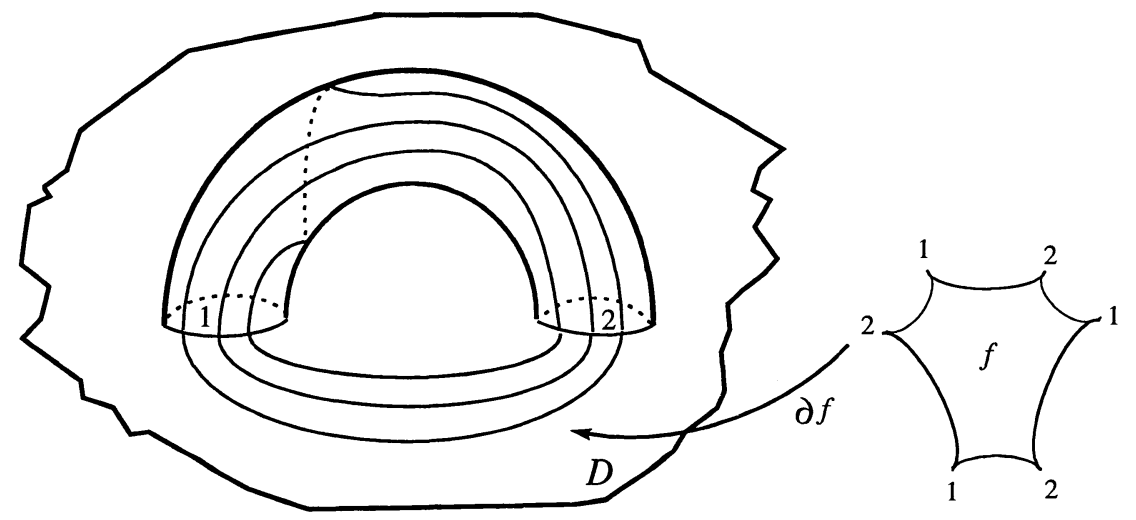

Figure 3.2.

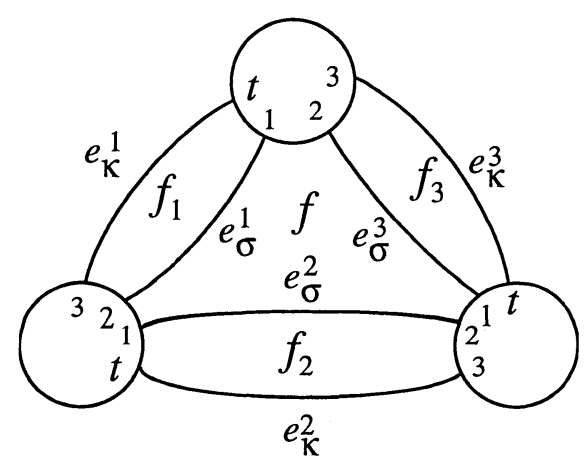

Figure 3.3.

Proof. Assume for contradiction that $\kappa$ is an extended Scharlemann cycle in $G_{Q}$. Let $\sigma$ be the Scharlemann cycle interior to $\kappa$. We may assume that the labels of the edges of $\sigma$ are $\{1,2\}$ and, consequently, the labels of the edges of $\kappa$ are $\{t, 3\}$. See Figure 3.3.

Without loss of generality, we assume that $H_{23}, H_{t 1}$ are in $X^{\prime}$ and $H_{12}$ is in $X$. Let $e_{\sigma}^{1}, e_{\sigma}^{2}, \ldots, e_{\sigma}^{k}$ be the edges of $\sigma$ and $e_{\kappa}^{1}, e_{\kappa}^{2}, \ldots, e_{\kappa}^{k}$ be the corresponding, parallel edges of $\kappa$. Let $f_{i}, i=1, \ldots, k$, be the bigon faces of $G_{Q}$ representing the parallelism of $e_{\sigma}^{i}$ with $e_{\kappa}^{i}$. Let $f$ be the face bounded by $\sigma$. See Figure 3.3.

Claim 3.3. $e_{\sigma}^{i} \cup e_{\sigma}^{j}$ lies in a disk in $\widehat{T}$ if and only if $e_{\kappa}^{i} \cup e_{\kappa}^{j}$ lies in a disk in $\widehat{T}$. 
Proof. Assume $e_{\sigma}^{i} \cup e_{\sigma}^{j}$ lies in a disk in $\widehat{T}$ and $e_{\kappa}^{i} \cup e_{\kappa}^{j}$ does not, i.e., $e_{\kappa}^{i} \cup e_{\kappa}^{j}$ forms an essential curve on $\widehat{T}$. Let $A$ be the annulus obtained by taking $H_{23} \cup H_{t 1} \cup f_{i} \cup f_{j}$ and shrinking $H_{23}$ and $H_{t 1}$ radially to their cores. The boundary of $A$ consists of two curves on $\widehat{T}$ : that formed by $e_{\sigma}^{i} \cup e_{\sigma}^{j}$ and that formed by $e_{\kappa}^{i} \cup e_{\kappa}^{j}$. By hypothesis the first component of $\partial A$ may be capped off with a disk, giving a compressing disk for $\widehat{T}$ in $K(\gamma)$. But $\widehat{T}$ is incompressible. This contradiction proves one direction of the claim, the other direction follows from the same argument.

Claim 3.3 and Lemma 3.1 imply that the edges of $\sigma$ lie in an annulus $A_{1}$ in $\widehat{T}$ and the edges of $\kappa$ lie in an annulus $A_{2}$ in $\widehat{T}$, such that $A_{1} \cap A_{2}=\emptyset$. Let $M_{1}=\operatorname{nhd}\left(A_{1} \cup H_{12} \cup f\right)$.

Claim 3.4. $M_{1}$ is a solid torus.

Proof. Assume for contradiction that $M_{1}$ is not a solid torus. $K(\gamma)$ is irreducible by [GLu4], thus $\partial M_{1}$ is an incompressible torus in $K(\gamma)$ (the incompressibility of $\widehat{T}$ guarantees that any compressing disk for $\partial M_{1}$ in $K(\gamma)$ must lie in $\left.M_{1}\right)$. Thus $\left|\partial M_{1} \cap K_{\gamma}\right|=2\left|A_{1} \cap K_{\gamma}\right|-2 \geq t$. Thus $\left|A_{1} \cap K_{\gamma}\right| \geq \frac{t}{2}+1$. Thus $\left|A_{2} \cap K_{\gamma}\right|<\frac{t}{2}$. Let $F$ be the disk in $Q$ bounded by $\kappa$ (containing $\sigma$ ). Let $H_{t 3}=H_{t 1} \cup H_{12} \cup H_{23}$ and $N=\operatorname{nhd}\left(A_{2} \cup H_{t 3} \cup F\right)$. Then Claim 3.3 and the irreducibility of $K(\gamma)$ guarantee that $N$ is isotopic to $M_{1}\left(e_{\sigma}^{i}\right.$ and $e_{\sigma}^{j}$ parallel on $A_{1}$ implies that $e_{\kappa}^{i}$ and $e_{\kappa}^{j}$ are parallel on $A_{2}$ - so $f_{i}$ and $f_{j}$ are parallel in $X^{\prime}$ ). In particular, $\partial N$ is an essential torus in $K(\gamma)$. But $\left|\partial N \cap K_{\gamma}\right|=2\left|A_{2} \cap K_{\gamma}\right|-2<t-2<t$. This contradicts the minimality of $\left|\widehat{T} \cap K_{\gamma}\right|$.

Now $\partial M_{1}=A_{1} \cup B$ where $B$ is an annulus properly embedded in $X$. Then $T_{2}=B \cup\left(\widehat{T}-A_{1}\right)$ is a torus which $K_{\gamma}$ intersects fewer than $t$ times. Thus $T_{2}$ bounds a solid torus $M_{2}$ (again using the irreducibility of $K(\gamma)$ ) and $X=M_{1} \cup_{B} M_{2}$. Since $\widehat{T}$ is incompressible in $K(\gamma), B$ must be homotopic to at least twice around the cores of both $M_{1}$ and $M_{2}$. This means that $X$ is a Seifert fiber space over a disk with two exceptional fibers, and the Seifert fiber is given by the core of $B$.

Let $e_{\sigma}^{i}$ and $e_{\sigma}^{j}$ be edges of $\sigma$ which form an essential simple closed curve on $\widehat{T}$ and have the property that they are outermost on $A_{1}$ among all such edges, i.e., on one side of $e_{\sigma}^{i} \cup e_{\sigma}^{j}$ in $A_{1}$ there are no further edges of $\sigma$. We may assume (by possibly taking a smaller $A_{1}$ ), that there are no vertices of $G_{T}$ on this side of $e_{\sigma}^{i} \cup e_{\sigma}^{j}$ in $A_{1}$. See Figure 3.4. 


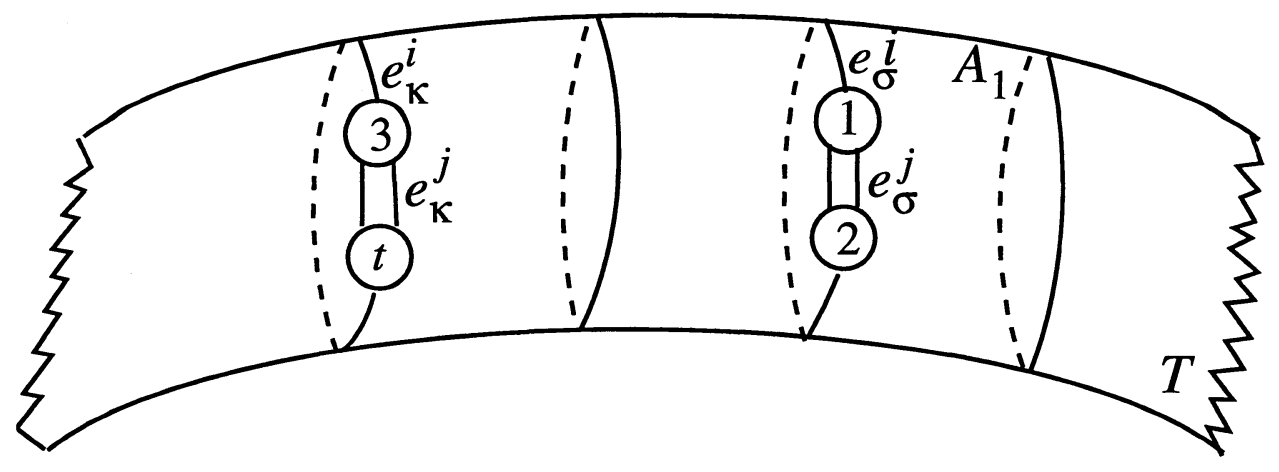

Figure 3.4.

Let $A$ be the annulus obtained by taking $H_{23} \cup H_{t 1} \cup f_{i} \cup f_{j}$ and shrinking $H_{23}$ and $H_{t 1}$ radially to their cores. The boundary of $A$ consists of two curves $e_{\sigma}^{i} \cup e_{\sigma}^{j}$ and $e_{\kappa}^{i} \cup e_{\kappa}^{j}$. By Claim 3.3 both of these curves are essential on $\widehat{T}$. They therefore divide $\widehat{T}$ into two annuli, $C_{1}$ and $C_{2}$. Since $K(\gamma)$ does not contain a Klein bottle, by Theorem 1.3, $A \cup C_{1}$ and $A \cup C_{2}$ are tori, $\widehat{T}_{3}$ and $\widehat{T}_{4}$, say.

Each of $\widehat{T}_{3}$ and $\widehat{T}_{4}$ intersects $K_{\gamma}$ fewer times than $\widehat{T}$ (after pushing $K_{\gamma}$ off of $A$ in the right direction); consequently, by the minimality of $\widehat{T}$ and the irreducibility of $K(\gamma), \widehat{T}_{3}, \widehat{T}_{4}$ bound solid tori, $M_{3}, M_{4}$. Thus $X^{\prime}=$ $M_{3} \cup_{A} M_{4}$. The incompressibility of $\widehat{T}$ implies that $A$ must be homotopic to at least twice around the core in each of $M_{3}$ and $M_{4}$; therefore, $X^{\prime}$ is a Seifert fiber space over the disk with two exceptional fibers and the Seifert fiber is represented by the core of $A$.

After enlarging by an isotopy in $X^{\prime}$, we may assume that, say, $C_{1}$ contains $A_{1}$ (note that $A$ can still be taken disjoint from $K_{\gamma}$ ). Let $Y=M_{3} \cup_{A_{1}} M_{1}$. The core of $A_{1}$ is isotopic to the core of $A$ and to the core of $B$. Thus the core of $A_{1}$ is homotopic to at least twice around the core in each of $M_{3}$ and $M_{1}$. Thus $Y$ is a Seifert fiber space over the disk with two exceptional fibers. Let $Y^{\prime}=K(\gamma)-\operatorname{Int} Y=M_{2} \cup_{C_{2}} M_{4}$. Since $C_{2}$ is homotopic to at least twice around the cores of $M_{2}$ and $M_{4}, Y^{\prime}$ is also a Seifert fiber space over the disk with two exceptional fibers. Therefore $\partial Y$ is incompressible in $K(\gamma)$. But $\left|\partial Y \cap K_{\gamma}\right|=\left|\left(C_{1}-A_{1}\right) \cap K_{\gamma}\right|+\left|B \cap K_{\gamma}\right|<\left|C_{1} \cap K_{\gamma}\right| \leq t$, contradicting the minimality of $\widehat{T}$.

(Theorem 3.2)

Theorem 3.5. There can be at most two Scharlemann cycles of $G_{Q}$ on distinct label-pairs whose faces lie on the same side of $\widehat{T}$. Furthermore, if 
there are two then this side of $\widehat{T}$ is a Seifert fiber space over the disk with two exceptional fibers whose orders are equal to the lengths of these Scharlemann cycles.

Proof. Assume there are two Scharlemann cycles $\sigma_{1}, \sigma_{2}$ and possibly a third $\sigma_{3}$ on distinct label-pairs that lie in, say, $X$. Let $f_{1}, f_{2}, f_{3}$ be the faces of $G_{Q}$ bounded by $\sigma_{1}, \sigma_{2}, \sigma_{3}$ (resp.). Then the boundaries of these faces lie on disjoint annuli $A_{1}, A_{2}, A_{3}$ in $\widehat{T}$. Let $\left\{k_{i}, k_{i}+1\right\}$ be the labels of $\sigma_{i}$. Define $M_{i}=\operatorname{nhd}\left(A_{i} \cup H_{k_{i}, k_{i}+1} \cup f_{i}\right)$.

Claim 3.6. If $M_{i}$ is a solid torus then the core of $A_{i}$ must be homotopic to $n_{i}$ times a generator of $\pi_{1}\left(M_{i}\right)$, where $n_{i}$ is the length of $f_{i}$.

Proof. Attach a 2-handle to $M_{i}$ along the core of $A_{i}$. From the handle decomposition one sees that the resulting manifold has first homology of order $n_{i}$.

For $i=1,2$, let $Z_{i}=\overline{X-M_{i}}$. Then $\left|\partial Z_{i} \cap K_{\gamma}\right|<t$. Thus $\partial Z_{i}$ must compress in $K(\gamma)$. Hence it must compress in $Z_{i}$, by the incompressibility of $\widehat{T}$. This fact along with the irreducibility of $K(\gamma)$ implies that $Z_{i}$ is a solid torus.

Let $Z_{12}=Z_{1} \cap Z_{2}$. Writing $\partial M_{2}=A_{2} \cup B_{2}$, we have $Z_{1}=Z_{12} \cup_{B_{2}} M_{2}$. This means that $Z_{12}$ and $M_{2}$ are both solid tori and that the core of $B_{2}$ is isotopic to the core of one of these solid tori. By Claim 3.6, it must be isotopic to the core of $Z_{12}$. This implies the theorem. First, there cannot be a third Scharlemann cycle $\sigma_{3}$. For then $M_{3} \subset Z_{12}$, but this contradicts Claim 3.6. Second, it expresses $X$ as a Seifert fiber space over a disk with two exceptional fibers, where Claim 3.6 says that the orders of these fibers are the lengths of $\sigma_{1}, \sigma_{2}$.

(Theorem 3.5)

Lemma 3.7. Let $\sigma$ be a Scharlemann cycle in $G_{Q}$ of length $n$, where $n$ is either 2 or 3 . Then the edges of $\sigma$ lie in an essential annulus, $A$, in $\widehat{T}$. Let $\{k, k+1\}$ be the labels of $\sigma$ and let $f$ be the face of $G_{Q}$ bounded by $\sigma$. Then $M=\operatorname{nhd}\left(A \cup H_{k, k+1} \cup f\right)$ is a solid torus such that the core of $A$ is homotopic in $M$ to $n$ times around the core of $M$.

Proof. The only way that the edges can fail to lie on an essential annulus in $\widehat{T}$ is if $\sigma$ has length 3. By Lemma 3.1, the edges on $G_{T}$ and $G_{Q}$ would be 

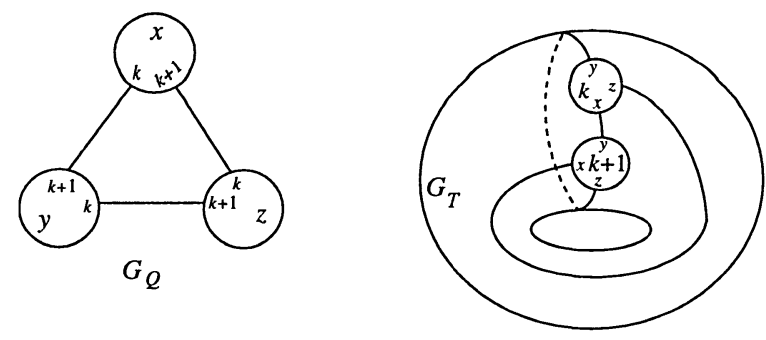

Figure 3.5.

as in Figure 3.5. But because $\widehat{T}$ is separating, the clockwise orientation of labels $x, y, z$ on vertex $k$ would force an anticlockwise orientation of $x, y, z$ on vertex $k+1$, contradicting Figure 3.5.

$M$ is a genus two handlebody, $A \cup H_{k, k+1}$, with a 2-handle, $f$, attached. Let $m_{1}$ be a cocore of $H_{k, k+1}$ that intersects $\partial f$ algebraically and geometrically $n$ times. Let $m_{2}$ be a meridianal disk of $\operatorname{nhd}(A)$ that intersects $\partial f$ once (Lemma 3.1). Then $m_{1}, m_{2}$ form a set of meridianal disks for the handlebody. Since $\partial f$ intersects $m_{2}$ once, $\partial f$ is primitive and $M$ is a solid torus. Since the core of $A$ intersects $m_{2}$ once and misses $m_{1}$, the core of $A$ is homotopic to $n$ times the core of $M$.

Theorem 3.8. Let $\sigma, n, A, f$ be as in Lemma 3.7. The side of $\widehat{T}$ containing $f$ is a Seifert fiber space over the disk with two exceptional fibers one of which has order $n$. Furthermore, the core of $A$ is a Seifert fiber.

Proof. Let $M$ be as in Lemma 3.7 and assume without loss of generality that $f$ is in $X$. We can write $\partial M=A \cup B$ where $B$ is an annulus properly embedded in $X$. Let $\widehat{T}^{\prime}=(\widehat{T}-A) \cup B$. $\widehat{T}^{\prime}$ intersects $K(\gamma)$ fewer times than $\widehat{T}$, hence must compress in $K(\gamma)$. By the incompressibility of $\widehat{T}$, this compressing disk must lie in $X$. Thus $\widehat{T}^{\prime}$ bounds a solid torus $M^{\prime}$ and $X=M \cup_{B} M^{\prime}$. By Lemma $3.7, M$ is a solid torus. Since $\widehat{T}$ is incompressible, $X$ is a Seifert fiber space over the disk with two exceptional fibers given by the cores of $M$ and $M^{\prime}$. Furthermore, the core of $B$ is a Seifert fiber, hence so is the core of $A$. By Lemma 3.7 the core of $M$ represents an exceptional fiber of order $n$.

Theorem 3.9. Let $\sigma_{1}$ and $\sigma_{2}$ be Scharlemann cycles in $G_{Q}$ of order 2 or 3 and let $f_{1}$ and $f_{2}$ be the faces of $G_{Q}$ that they bound. If $f_{1}$ and $f_{2}$ lie on opposite sides of $\widehat{T}$, then $\sigma_{1}$ and $\sigma_{2}$ must have a common label. 
Proof. Assume for contradiction that $\sigma_{1}$ and $\sigma_{2}$ do not have a common label. Then the edges of $\sigma_{1}$ and $\sigma_{2}$ lie on disjoint annuli in $\widehat{T}, A_{1}$ and $A_{2}$ (resp.). Assume $f_{1}$ lies in $X$ and $f_{2}$ lies in $X^{\prime}$. Let $M_{i}=\operatorname{nhd}\left(A_{i} \cup H_{k_{i}, k_{i}+1} \cup f_{i}\right)$ for $i=1,2$ where $k_{i}, k_{i}+1$ are the labels of $\sigma_{i}$. Let $B_{i}=\partial M_{i}-A_{i}, i=1,2$. Then $B_{1}$ is a properly embedded annulus in $X$ and $B_{2}$ is a properly embedded annulus in $X^{\prime}$. The argument of Theorem 3.8 shows that $M_{1}^{\prime}=\overline{X-M_{1}}$ is a solid torus for which the core of $B_{1}$ is homotopic to more than once around the core of $M_{1}^{\prime}$. Similarly the core of $B_{2}$ is homotopic to more than once around the core of $M_{2}^{\prime}=\overline{X^{\prime}-M_{2}}$. Let $\widehat{T}^{\prime}=\left(\widehat{T}-\left(A_{1} \cup A_{2}\right)\right) \cup B_{1} \cup B_{2}$. Note that $\widehat{T}^{\prime}$ intersects $K_{\gamma}$ fewer times than $\widehat{T}$. One side of $\widehat{T}^{\prime}$ is $N_{1}=M_{1} \cup_{A_{1}} M_{2}^{\prime}$ and the other side is $N_{2}=M_{2} \cup_{A_{2}} M_{1}^{\prime} . \quad N_{1}$ is a Seifert fiber space over the disk with two exceptional fibers since the core of $A_{1}$ is homotopic to a proper multiple of the cores of both $M_{1}$ and $M_{2}^{\prime}$ (the core of $A_{1}$ is isotopic to the core of $A_{2}$, hence also to the core of $B_{2}$ ). Similarly, $N_{2}$ is a Seifert fiber space over the disk with two exceptional fibers. Thus $\widehat{T}^{\prime}$ is incompressible in $K(\gamma)$. But this contradicts the minimality of $\widehat{T}$.

Note. By Theorem 3.8, both $X$ and $X^{\prime}$ are Seifert fibered over the disk with two exceptional fibers; furthermore, the Seifert fibers are isotopic on $\widehat{T}$. Thus $K(\gamma)$ is Seifert fibered over the 2-sphere with four exceptional fibers. In the above argument, $\widehat{T}$, which separates the exceptional fibers into pairs, is exchanged for $\widehat{T}^{\prime}$ which separates the fibers into different pairs.

Lemma 3.10. Let $\sigma_{1}$ and $\sigma_{2}$ be Scharlemann cycles of length 2 in $G_{Q}$ on distinct label-pairs. Then the loops on $\widehat{T}$ formed by the edges of $\sigma_{1}$ and $\sigma_{2}$ respectively are not isotopic on $\widehat{T}$.

Proof. Let $\left\{k_{i}, k_{i}+1\right\}$ be the labels of $\sigma_{i}$, and let $f_{i}$ be the face of $G_{Q}$ bounded by $\sigma_{i}, i=1,2$. Then shrinking $H_{k_{i}, k_{i}+1}$ to its core in $H_{k_{i}, k_{i}+1} \cup f_{i}$ gives a Möbius band $B_{i}$ such that $\partial B_{i}$ is the loop on $\widehat{T}$ formed by the edges of $\sigma_{i}$. If $\partial B_{1}$ were isotopic to $\partial B_{2}$ on $\widehat{T}$ then taking the union of $B_{1}$ and $B_{2}$ would give a Klein bottle in $K(\gamma)$, contradicting Theorem 1.3.

Theorem 3.11. At most three labels can be labels of Scharlemann cycles of length 2 in $G_{Q}$. 
Proof. If there are four such labels, then there are two Scharlemann cycles of length 2, $\sigma_{1}$ and $\sigma_{2}$, say, whose label-pairs are disjoint. By Lemma 3.1, the loops on $\widehat{T}$ formed by the edges of $\sigma_{1}$ and $\sigma_{2}$ are isotopic. But this contradicts Lemma 3.10.

\section{The case $\Delta \geq 3, t \geq 4$.}

In this section we prove the following weak version of Theorem 1.1. As mentioned at the end of Section 1, Theorem 4.1, Theorem 8.1 and the main result of [EM1] imply Theorem 1.1.

Theorem 4.1. Suppose that $K(\gamma)$ contains an incompressible torus, where $\Delta(\gamma, \mu) \geq 3$. Then $t=2$.

Let $\Lambda$ be a great web in $G_{Q}$ guaranteed by Corollary 2.7. Let $D_{\Lambda}$ be the disk bounded by $\Lambda$.

For every label $x$ of $G_{Q}$ let $\Lambda_{x}$ be the graph in $D_{\Lambda}$ consisting of the vertices of $\Lambda$ along with all $x$-edges of $\Lambda$. Note that, by the parity rule, an edge of $\Lambda$ has at most one end with label $x$.

Let $V$ be the number of vertices of $\Lambda$. Recall that $V>1$.

Lemma 4.2. Let $x$ be a label of $G_{Q}$. If $\Lambda_{x}$ has at least $3 V-4$ edges, then $\Lambda_{x}$ contains a bigon.

Proof. Assume that $\Lambda_{x}$ contains at least $3 V-4$ edges. We may assume that $\Lambda_{x}$ is connected. We will view $\Lambda_{x}$ as a graph in the 2-sphere, where at most one face $f$ of $\Lambda_{x}$ contains vertices of $G_{Q}$ that are not in $\Lambda_{x}$. We assume for contradiction that the only bigon of $\Lambda_{x}$ is possibly $f$. Let $V, E, F$ be the number of vertices, edges, and faces of $\Lambda_{x}$ considered as a graph in the 2-sphere. Then by counting edges we have $2+3(F-1) \leq 2 E$ which implies that $F \leq(2 E+1) / 3$. We also have $E \geq 3 V-4$. Combining this with the Euler characteristic equation $V-E+F=2$ we obtain

$$
(E / 3+4 / 3)-E+(2 E+1) / 3 \geq 2
$$

But this is a contradiction.

Let $\mathcal{L}$ be the set of all labels of $G_{Q}$ that are labels of Scharlemann cycles of length 2 in $G_{Q}$. 
Theorem 4.3. If $\Delta \geq 3$ then $|\mathcal{L}| \geq 4 t / 5$.

Proof. Let $x$ be a label such that the number of edges of $\Lambda_{x}$ is at least $3 V-4$. Applying Lemma 4.2, $\Lambda_{x}$ must contain a bigon. But such a bigon is either the face of $G_{Q}$ bounded by a Scharlemann cycle of length 2, or contains an extended Scharlemann cycle of length 2. (Note that the $x$-labels of the two edges cannot appear on the same corner of the bigon, else there would be $t$ edges of $\Lambda$ within this bigon and, consequently, since no edge of $\Lambda$ has the same label at both ends, there would be another edge of $\Lambda_{x}$ within the bigon.) Hence, by Theorem $3.2, x \in \mathcal{L}$.

Thus if $x \notin \mathcal{L}$ then there can be at most $3 V-5$ edges in $\Lambda_{x}$. Since $\Delta \geq 3$, the label $x$ appears at least three times at each vertex of $G_{Q}$. Thus if $x \notin \mathcal{L}$ there must be at least five occurrences of label $x$ where edges of $G_{Q}$ that are not in $\Lambda$ are incident to $\Lambda$ (i.e., ghost $x$-labels). By the definition of a web, $\Lambda$ has at most $t$ ghost labels in all. Therefore, there can be at most $t / 5$ labels of $G_{Q}$ not in $\mathcal{L}$. Since $G_{Q}$ has $t$ labels, this proves the theorem.

Proof of Theorem 4.1. Suppose $t \geq 4$. Then Theorem 4.3 implies that $|\mathcal{L}| \geq 4$. But $|\mathcal{L}| \leq 3$ by Theorem 3.11 .

\section{The case $\Delta=2, t \geq 6$.}

In this section we prove Theorem $1.2^{\prime}$, which we restate here for the reader's convenience.

Theorem 1.2'. Suppose that $K(\gamma)$ contains an incompressible torus, where $\Delta(\gamma, \mu)=2$. Then $t=2$ or 4 .

Recall that $\Lambda$ is a great web guaranteed by Corollary 2.7. In what follows we assume for contradiction that $t \geq 6$ and derive a sequence of lemmas describing $\Lambda$. These lemmas come together in a proof of Theorem $1.2^{\prime}$ at the end of the section.

As in Section 4, for each label $x$ of $G_{Q}$ let $\Lambda_{x}$ be the subgraph of $\Lambda$ consisting of all the vertices of $\Lambda$ together with all $x$-edges in $\Lambda$. We assume without loss of generality that the labels appear in anticlockwise order around the vertices of $\Lambda$. 
Lemma 5.1. A face of $\Lambda_{x}$ of length at most 3 is either a face bounded by a Scharlemann cycle or one of the faces illustrated in Figure 5.1 (a)-(f).

Proof. It is clear that the boundary of a face of $\Lambda_{x}$ of length 2 is either a Scharlemann cycle or an extended Scharlemann cycle. But the latter is impossible by Theorem 3.2 .

So let $h$ be a face of $\Lambda_{x}$ of length 3 . The argument now breaks up into two cases, according to whether or not the edges of $h$ form an $x$-cycle.

\section{Case I. The edges of $h$ form an $x$-cycle.}

It is easy to see that $h$ contains within it a Scharlemann cycle of length 2 or 3 . If this Scharlemann cycle is of length 3 then $h$ is either a Scharlemann cycle or an extended Scharlemann cycle, and hence must be a Scharlemann cycle. We may assume, then, that $h$ contains within it an $(r, r+1)$ Scharlemann cycle of length 2 . Since $G_{Q}$ contains no extended Scharlemann cycles of length 2, within $h$ we see the configuration of either Figure 5.2(i) or Figure 5.2(ii). We consider Figure 5.2(i); the case of Figure 5.2(ii) is similar.

Let $I$ and $J$ be the label-intervals indicated at vertex $c$. Since $|I|$ is equal to the number of labels between $r+2$ and $x$ (at vertex $a$ ), we see that $r+2 \in I \cup J$.

If $r+2 \in I$, then among the edges joining $a$ and $c$ we have either an extended Scharlemann cycle or an $(r+2, r+3)$-Scharlemann cycle of length 2, contradicting Theorem 3.2 or Theorem 3.11 respectively. Hence $r+2 \in J$.

Similarly, if $w<r$, then (by the parity rule) $w \leq r-2$, and hence $r-1 \in J$, giving either an extended Scharlemann cycle or an $(r-2, r-1)-$ Scharlemann cycle of length 2 among the edges joining $b$ and $c$. Thus $w=r$ or $r+2$, and we have either Figure 5.1(a) or Figure 5.1(b).

A similar argument shows that Figure 5.2(ii) gives rise to either Figure 5.1(c) or Figure 5.1(d).

\section{Case II. The edges of $h$ do not form an $x$-cycle.}

Let the vertices of $h$ be $a, b, c$, where $a$ is the vertex at which both edges of $h$ incident to $a$ have label $x$. Let $e(a, b)$ and $e(a, c)$ be the number of edges within $h$ joining $a$ to $b$ and $c$ respectively. Since $e(a, b)+e(a, c)=t+1$, we have (say) $e(a, b)>t / 2$. We therefore get a length 2 Scharlemann cycle among these edges. By Theorems 3.2 and 3.11, we must have $e(a, b)=$ $t / 2+1$, and also an $(r, r+1)$-Scharlemann cycle must occur at the end of the group of parallel edges. Thus the situation is as illustrated in Figure 5.3 , with $u=x-1$. Also, $e(a, c)=t / 2$. Since by Theorem 3.11 there 
Dehn Surgeries on Knots

617

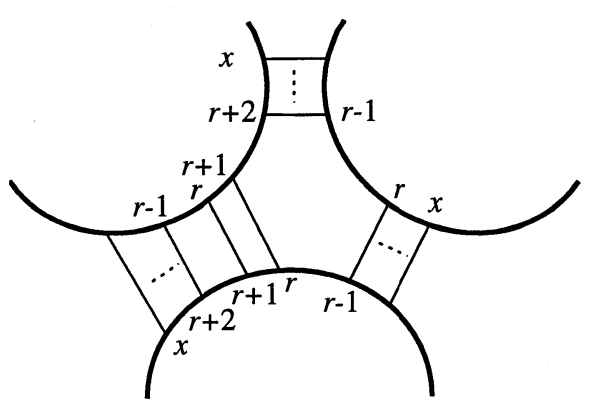

(a)

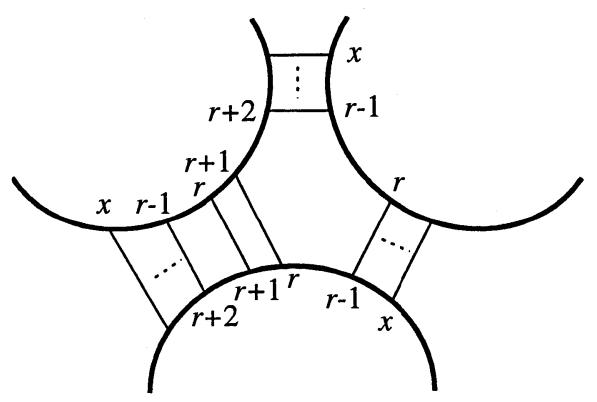

(c)

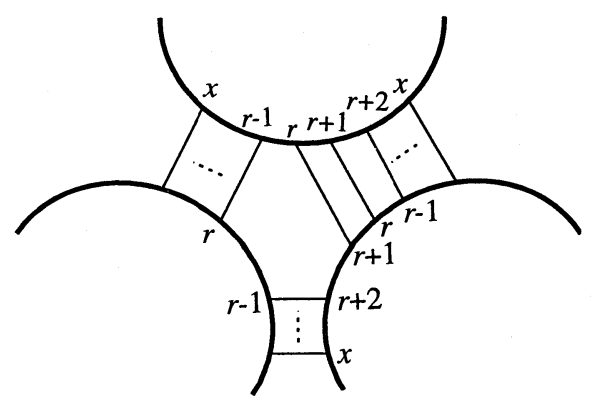

(e)

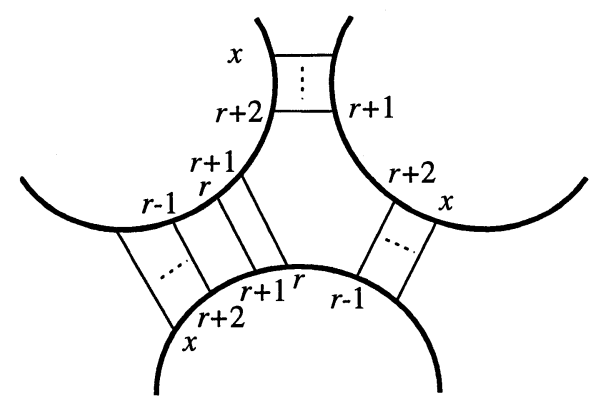

(b)

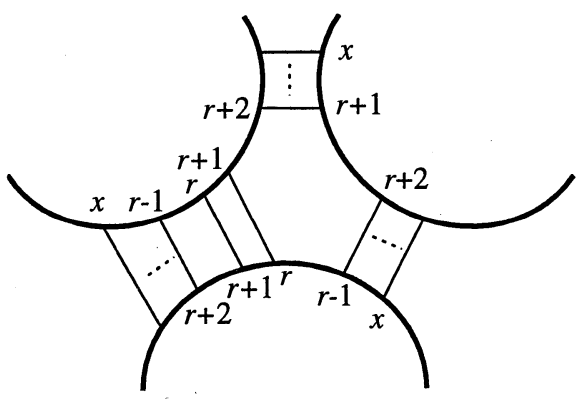

(d)

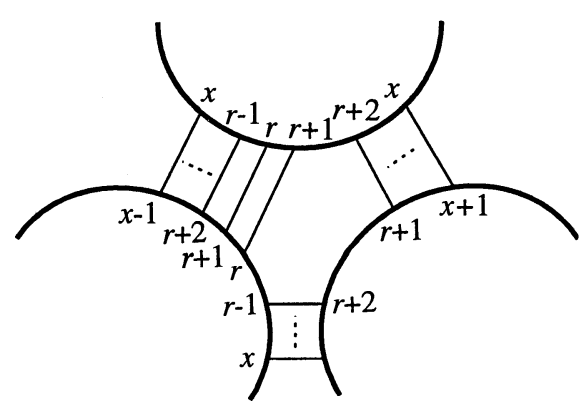

(f)

Figure 5.1. 


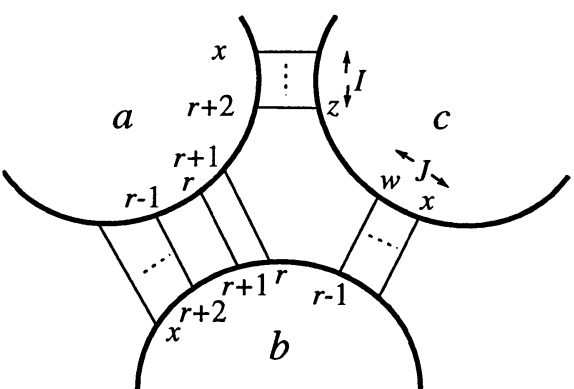

(i)

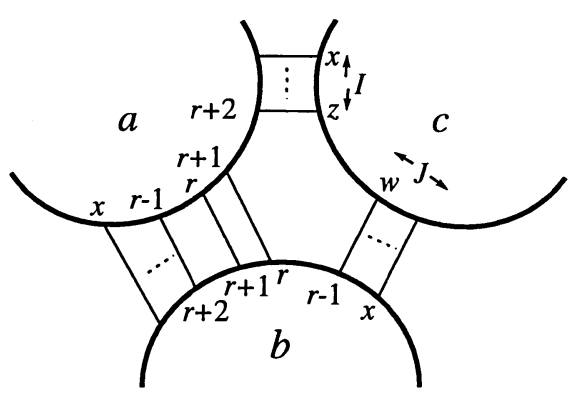

(ii)

Figure 5.2.

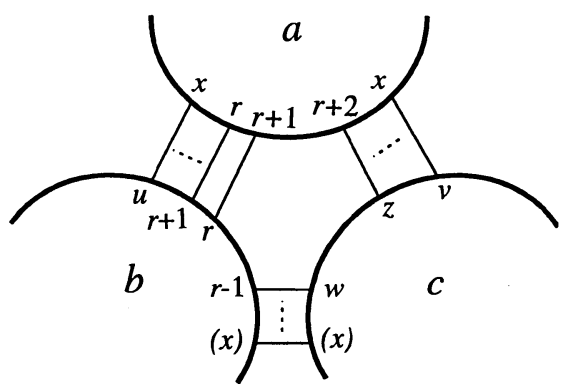

Figure 5.3.

is no Scharlemann cycle among the edges joining $a$ and $c$, we must have $v=x+1, z=r+1$, and $w=r+2$. Finally we note that the label $x$ appears on vertex $b$ before vertex $c$ in Figure 5.3, giving us Figure 5.1(f). Similarly, if $e(a, c)>t / 2$ we get Figure 5.1(e).

Lemma 5.1 shows that if $\Lambda_{x}$ contains a face of length at most 3 whose boundary is not a Scharlemann cycle then $G_{Q}$ contains one of the configurations $C_{ \pm}(r)$ illustrated in Figure 5.4.

Lemma 5.2. Suppose that $G_{Q}$ contains one of the configurations $C_{ \pm}(r)$. Then

(1) the edges in $C_{ \pm}(r)$ lie on $\widehat{T}$ as shown in Figure 5.5.

Furthermore, if $A$ is an annulus in $\widehat{T}$ containing these edges then

(2) $\left|A \cap K_{\gamma}\right| \geq t / 2+2$; and 


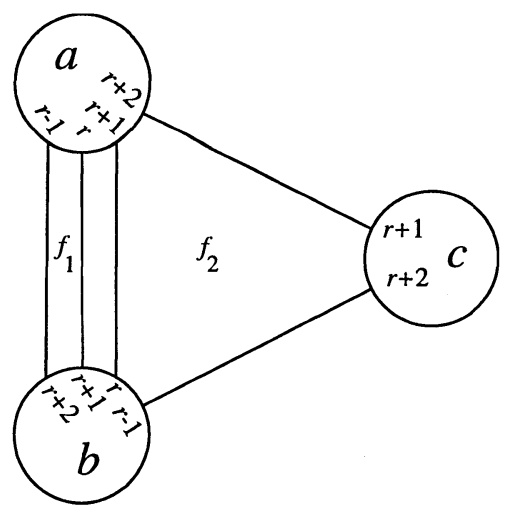

$C_{+}(r)$

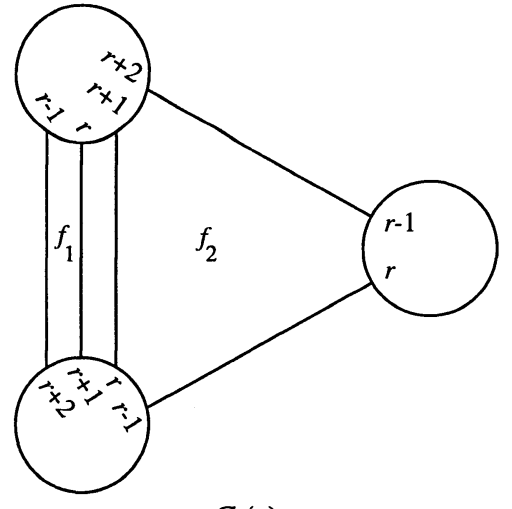

$C_{-}(r)$

Figure 5.4.

(3) $N=\operatorname{nhd}\left(A \cup H_{r-1, r} \cup H_{r+1, r+2} \cup f_{1} \cup f_{2}\right)$ is a Seifert fiber space over the disk with two exceptional fibers of orders 2 and 3.

Proof. Assume $G_{Q}$ contains $C(r)=C_{+}(r)$; the case of $C_{-}(r)$ is completely analogous.

Since the two $(r, r+1)$ edges in $C(r)$ form a Scharlemann cycle, they form an essential loop on $\widehat{T}$ by Lemma 3.1.

Assume that the two $(r-1, r+2)$ edges in $C(r)$ do not form an essential loop on $\widehat{T}$. Let $A$ be an annulus in $\widehat{T}$ containing the union $E(r)$ of the edges of $C(r)$. Let $f_{1}, f_{2}$ be the faces of $G_{Q}$ indicated in Figure 5.4, and let $N=\operatorname{nhd}\left(A \cup H_{r-1, r} \cup H_{r+1, r+2} \cup f_{1} \cup f_{2}\right)$. Then

$$
\pi_{1}(N)=\left\langle x, y, z \mid x y=1, y^{2} x z=1\right\rangle
$$

where $x, y$, and $z$ are represented by the cores of $H_{r-1, r}, H_{r+1, r+2}$, and $A$ respectively. Thus $\pi_{1}(N) \cong \mathbb{Z}$, generated by $z$, and in fact it is easy to see that $N$ is a solid torus. Let $\partial N=A \cup A^{\prime}$. Then $\widehat{T}^{\prime}=(\widehat{T}-A) \cup A^{\prime}$ is a torus isotopic to $\widehat{T}$ with $\left|\widehat{T}^{\prime} \cap K_{\gamma}\right|=t-4$, contradicting our choice of $\widehat{T}$.

Hence $E(r)$ lies on $\widehat{T}$ as shown in Figure 5.5. Note that we may assume that the $(r, r+1)$ edges and the $(r+1, r+2)$ edge appear as in the Figure. If $a, b, c$ are the vertices of Configuration $C_{+}(r)$ then the orientation of these labels at vertex $r+1$ forces the labeling at vertex $r+2$.

Define $A$ and $N$ as above. Now

$$
\pi_{1}(N)=\left\langle x, y, z \mid x y z^{-1}=1, y^{2} x z=1\right\rangle
$$




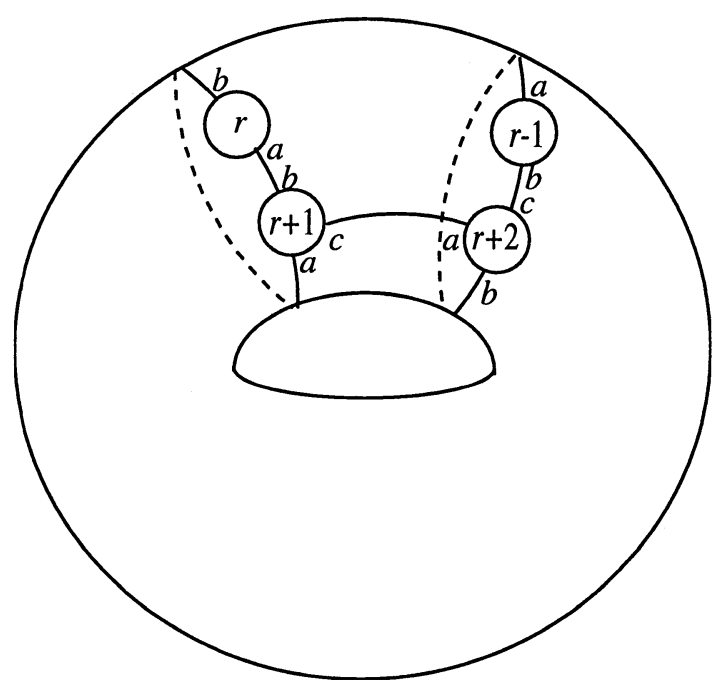

Figure 5.5.

$$
\cong\left\langle x, y \mid x^{2} y^{3}=1\right\rangle
$$

Hence $N$ is not a solid torus. In fact it is easy to see that $N$ is a Seifert fiber space over the disk with two exceptional fibers of orders 2 and 3 , in other words, the exterior of the trefoil knot. Therefore $\partial N$ is a torus which is incompressible in $N$, and in $K(\gamma)-\operatorname{Int} N$ (since $\widehat{T} \subset K(\gamma)-\operatorname{Int} N$ and $\widehat{T} \cap \partial N=A)$, and hence in $K(\gamma)$. If $\left|A \cap K_{\gamma}\right| \leq t / 2+1$, then $\mid \partial N \cap$ $K_{\gamma} \mid \leq 2(t / 2+1)-4=t-2$, contradicting the minimality of $t$. Hence $\left|A \cap K_{\gamma}\right| \geq t / 2+2$.

Lemma 5.3. Let $h$ be a face of $\Lambda_{x}$ as in Figure 5.1(a)-(f).

(1) If $x \in\{r-2, r+3\}$ then the two $(r-2, r+3)$ edges within $h$ form an essential loop on $\widehat{T}$.

(2) $x \in\{r-2, r-1, r+2, r+3\}$

Proof. If $x \in\{r-2, r+3\}$ then within $h$ we see Figure 5.6 (i) or (ii):

Let $B$ be the annulus formed from $g_{1} \cup g_{2} \cup H_{r-2, r-1} \cup H_{r+2, r+3}$ by shrinking the $H$ 's to their cores. By Lemma 5.2, the component of $\partial B$ formed by the $(r-1, r+2)$ edges of $g_{1}$ and $g_{2}$ is essential on $\widehat{T}$. Since $\widehat{T}$ is incompressible, the other component of $\partial B$ is also essential. This proves (1). 


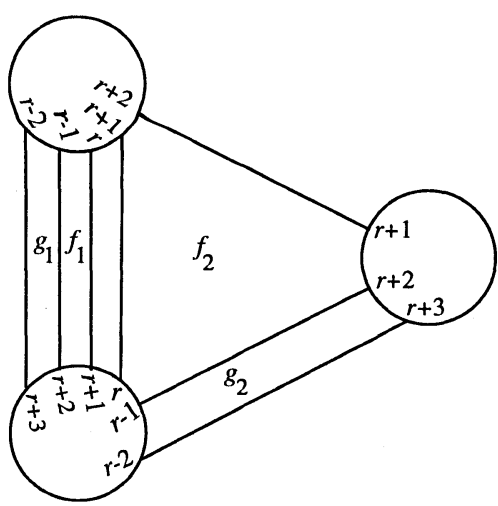

(i)

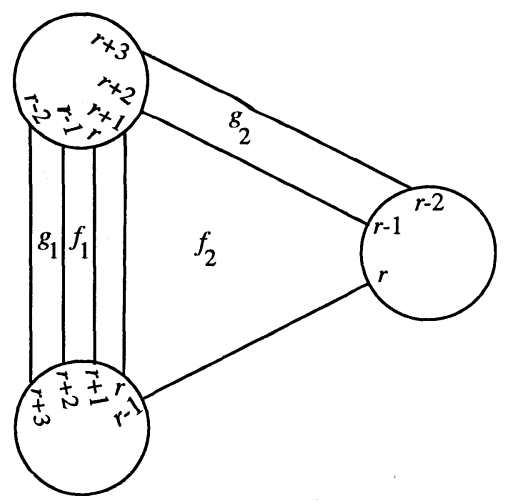

(ii)

Figure 5.6.
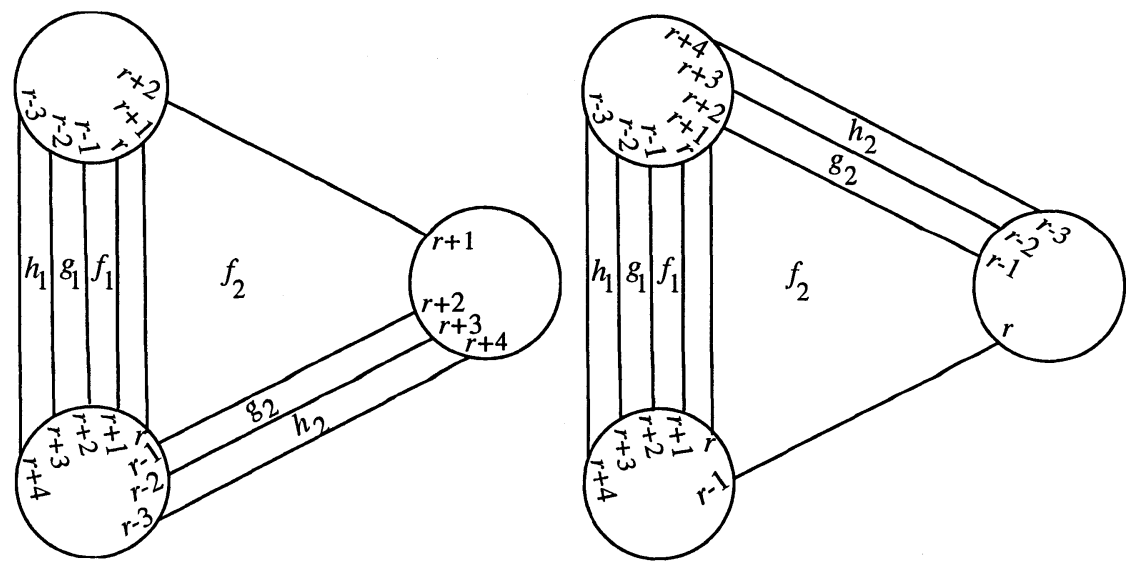

Figure 5.7.

To prove (2), observe that if $x \notin\{r-2, r-1, r+2, r+3\}$ then within $h$ we see one of the configurations of Figure 5.7

Let $c_{1}$ be the loop on $\widehat{T}$ formed by the $(r-2, r+3)$ edges of $h_{1}$ and $h_{2}$, and let $c_{2}$ be the loop formed by the $(r-3, r+4)$ edges of $h_{1}$ and $h_{2}$. Let $B$ be the annulus obtained from $h_{1} \cup h_{2} \cup H_{r-3, r-2} \cup H_{r+3, r+4}$ by shrinking the $H$ 's to their cores.

By the argument given for (1) above, $c_{1}$ is essential on $\widehat{T}$. Hence $c_{2}$ is also essential. Let $A_{1}$ and $A_{2}$ be the two annuli into which $c_{1}$ and $c_{2}$ divide $\widehat{T}$. Let $\widehat{T}_{i}=A_{i} \cup B, i=1,2$. Then, since $K(\gamma)$ contains no Klein bottle by Theorem 1.3, $\widehat{T}_{1}$ and $\widehat{T}_{2}$ are tori. Note also that (after a slight perturbation) $\left|\widehat{T}_{i} \cap K_{\gamma}\right|<t, i=1,2$. 
Now $\widehat{T}$ separates $K(\gamma)$ into $X$ and $X^{\prime}$; without loss of generality the faces $h_{1}, h_{2}, f_{1}, f_{2}$ lie in $X$. Then $B$ separates $X$ into $Y_{1}$ and $Y_{2}$, say, where $\widehat{T}_{i}=$ $\partial Y_{i}, i=1,2$. Without loss of generality the manifold $N$ of Lemma 5.2(3) is contained in $Y_{1}$. It follows that $\widehat{T}_{1}$ is incompressible in $K(\gamma)$, contradicting the minimality of $t$.

Lemma 5.4. If $t \geq 6$ then $G_{Q}$ can contain a configuration $C_{ \pm}(r)$ for at most one value of $r$.

Proof. Suppose $G_{Q}$ contains configurations $C(r)=C_{ \pm}(r), C(s)=C_{ \pm}(s)$, for $r \neq s$. Then $C(r)$ (resp. $C(s))$ contains an $(r, r+1)$ (resp. $(s, s+1)$ )Scharlemann cycle $\rho$ (resp. $\sigma$ ) of length 2 . Hence by Theorem 3.11, we may assume that $s=r+1$. Applying Lemma 5.2 to $C(r)$ and $C(r+1)$ gives pairs of $(r, r+1)$ edges, $(r-1, r+2)$ edges, $(r+1, r+2)$ edges, and $(r, r+3)$ edges, each of which forms an essential loop on $\widehat{T}$. Since $t \geq 6, r-1, r, r+1$, $r+2$, and $r+3$ are all distinct, and it is easy to see that these loops must therefore be isotopic on $\widehat{T}$. In particular, the loops formed by the edges of $\rho$ and $\sigma$ are isotopic on $\widehat{T}$. But this contradicts Lemma 3.10.

For each label $x$ of $G_{Q}$, let $\alpha_{x}$ be the number of ghost $x$-labels in $\Lambda$.

Theorem 5.5. If $\alpha_{x} \leq 4$ then $\Lambda_{x}$ contains a face of length 2 or 3 .

Proof. Let $\Gamma$ be an innermost connected component of $\Lambda_{x}$ with the least number of ghost $x$-labels. Let $\alpha$ be the number of ghost $x$-labels in $\Gamma$; so $\alpha \leq \alpha_{x} \leq 4$. Regard $\Gamma$ as a graph in the disk $D_{\Lambda}$, and let $v, e, f$ be the number of vertices, edges, and faces of $\Gamma$, respectively. Since $\Delta=2$ (and, by the parity rule, each edge of $\Lambda_{x}$ has only one label $x$ ), we have

$$
e=2 v-\alpha
$$

Let $n_{j}$ be the number of faces of $\Gamma$ of length $j$, and let $\omega$ be the number of edges of $\Gamma$ (counted with multiplicity) in the outside region of $\Gamma$, i.e., that containing $\partial D_{\Lambda}$. Then

$$
\begin{aligned}
2 e & =\sum j n_{j}+\omega \\
& \geq 4 f-n_{3}-2 n_{2}+\omega
\end{aligned}
$$




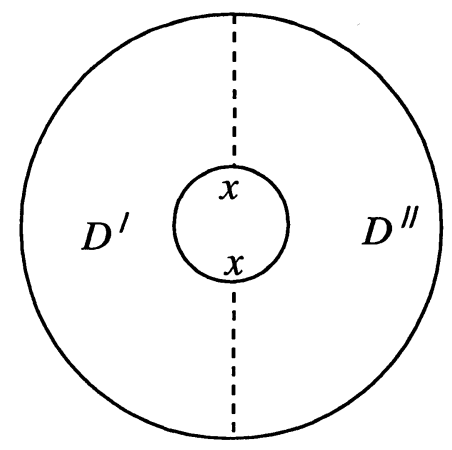

Figure 5.8.

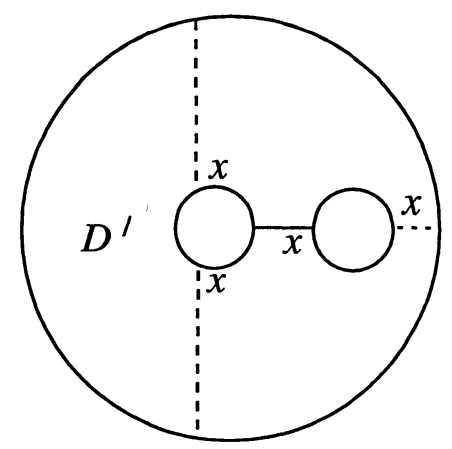

Figure 5.9.

Also, $1=v-e+f$. Hence

$$
\begin{aligned}
4=4 v-4 e+4 f & \leq 4 v-4 e+2 e+n_{3}+2 n_{2}-\omega \\
& =4 v-2(2 v-\alpha)+n_{3}+2 n_{2}-\omega \\
& =n_{3}+2 n_{2}-\omega+2 \alpha
\end{aligned}
$$

giving

$$
n_{3}+2 n_{2} \geq 4+\omega-2 \alpha .
$$

First observe that if $\omega=0$, then $\Gamma$ has a single vertex and is as shown in Figure 5.8.

Since the numbers of ghost labels of $\Lambda$ is at most $t$, there must be edges of $\Lambda$, and hence vertices of $\Lambda$, in both regions $D^{\prime}$ and $D^{\prime \prime}$. Hence there are components $\Gamma^{\prime}, \Gamma^{\prime \prime}$ of $\Lambda_{x}$ in $D^{\prime}, D^{\prime \prime}$ respectively. Since $\alpha_{x} \leq 4$, at least one of $\Gamma^{\prime}, \Gamma^{\prime \prime}$ has at most one ghost $x$-label, contradicting the minimality of $\Gamma$.

We may therefore assume that $\omega>0$. Then $(*)$ gives us the desired result unless $\alpha=3, \omega \leq 2$ or $\alpha=4, \omega \leq 4$.

Let $v_{0}$ be the number of vertices of $\Gamma$ in the outside region of $\Gamma$.

Case 1. $\alpha=3, \omega \leq 2$.

Since each vertex has at most two ghost $x$-labels, $v_{0} \geq 2$. Therefore $\omega=2$, and hence $v_{0}=2$. The situation is therefore as shown in Figure 5.9.

But then, as in the argument above that $\omega>0$, there is a component $\Gamma^{\prime}$ of $\Lambda_{x}$ in $D^{\prime}$ with at most one ghost $x$-label, contradicting the minimality of $\Gamma$. 


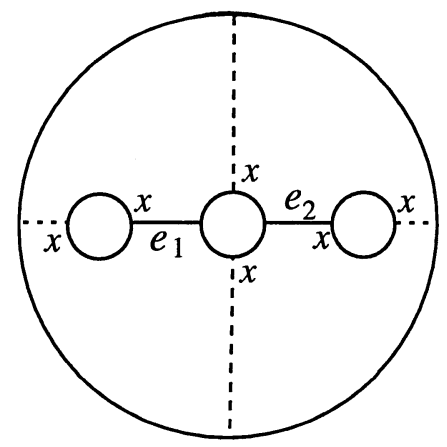

Figure 5.10.

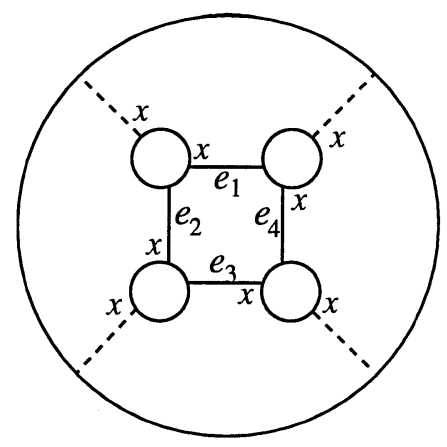

Figure 5.11.

Case 2. $\alpha=4, \omega \leq 4$.

First suppose there is a vertex of $\Gamma$ with two ghost $x$-labels, as in Figure 5.10. Then, as before, there must be vertices of $\Lambda$ in both regions $D^{\prime}$ and $D^{\prime \prime}$, and hence, by the minimality of $\Gamma, \Lambda$ and $\Gamma$ each have three vertices and $\Gamma$ is as shown in Figure 5.10.

If no vertex of $\Gamma$ has two ghost $x$-labels then $v_{0} \geq 4$. But $\omega \geq v_{0}$, hence $v_{0}=\omega=4$. Hence the outside region of $\Gamma$ is as shown in Figure 5.11. By the minimality of $\Gamma$ there are no vertices of $\Lambda$ in this outside region.

Now in both Figures 5.10 and 5.11, there can be at most $t / 2+1$ edges of $\Lambda$ parallel to each $e_{i}$, otherwise we would have either Scharlemann cycles of length 2 on disjoint label pairs, contradicting Theorem 3.11, or an extended Scharlemann cycle, contradicting Theorem 3.2.

Hence in Figure 5.10, each extremal vertex has at least $2 t-(t / 2+1)=$ $3 t / 2-1 \geq t$ ghost labels. Since the total number of ghost labels in $\Lambda$ is at most $t$, this is a contradiction.

Similarly, at each vertex in Figure 5.11 we see at least $t / 2$ ghost labels of $\Lambda$, giving a total of at least $2 t$ ghost labels, again a contradiction.

Theorem 5.6. For each label $x$ of $G_{Q}, \Lambda$ has at most t/2 ghost $x$-labels.

Proof. Since $\widehat{T}$ is separating any edge of $G_{Q}$ separates a black face of $G_{Q}$ from a white face. Thus as you move around the boundary of the outside region of $\Lambda$ (thought of as a graph in the disk $D_{\Lambda}$ ), the colors of the faces alternate as you cross the ghost labels. Since the vertices of $\Lambda$ are parallel this means that two consecutive ghost labels must correspond to vertices of 
$G_{T}$ which are not parallel. Since $\Lambda$ has at most $t$ ghost labels, we are done.

Let

$\mathcal{L}=\left\{x: \Lambda_{x}\right.$ contains a face of length 2 or 3$\}$, and

$\mathcal{S}=\left\{x: x\right.$ is a label of a Scharlemann cycle in $G_{Q}$ of length 2 or 3$\}$.

Let $\mathcal{L}_{0}=\mathcal{L}-\mathcal{S}$.

Theorem 5.7. $|\mathcal{S}| \leq 4$.

Proof. This is an immediate consequence of Theorems 3.5 and 3.9.

Theorem 5.8. $\left|\mathcal{L}_{0}\right| \leq 4$.

Proof. This follows from Lemma 5.1, Lemma 5.3(2) and Lemma 5.4.

Theorem 5.9. $|\mathcal{L}| \geq 4 t / 5$.

Proof. If $x \notin \mathcal{L}$ then by Theorem $5.5 \Lambda$ must contain at least five ghost $x$-labels. Since $\Lambda$ has at most $t$ ghost labels in total, the result follows.

Proof of Theorem $1.2^{\prime}$. Assume for contradiction that $t \geq 6$. Combining Theorems 5.7, 5.8 and 5.9 we get

$$
4 t / 5 \leq|\mathcal{L}|=\left|\mathcal{L}_{0}\right|+|\mathcal{S}| \leq 8 .
$$

Therefore $t \leq 10$.

Case 1. $t=10$ or 8 .

If $t=10$ then $|\mathcal{L}| \geq \frac{4 \cdot 10}{5}=8$ by Theorem 5.9.

If $t=8$ then by Theorem 5.6 we have $\alpha_{x} \leq 4$ for all labels $x$. Therefore $|\mathcal{L}|=8$ by Theorem 5.5 .

In both cases, then, we must have $\left|\mathcal{L}_{0}\right|=|\mathcal{S}|=4$.

In particular, $G_{Q}$ contains an $(r, r+1)$-Scharlemann cycle $\rho$ of length 2, and $\mathcal{L}_{0}=\{r-2, r-1, r+2, r+3\}$ by Lemma 5.3(2).

Since $|\mathcal{S}|>2, G_{Q}$ contains an $(s, s+1)$-Scharlemann cycle $\sigma$ of length 2 or 3 with $s \neq r$, and with $s, s+1 \notin \mathcal{L}_{0}$ (by definition of $\mathcal{L}_{0}$ ). Therefore $s, s+1 \notin\{r-2, r-1, r, r+1, r+2, r+3\}$. By Lemma 3.1, $\sigma$ contains a 
pair of $(s, s+1)$ edges that form an essential loop on $\widehat{T}$. Also, since $r-2$ (or $r+3) \in \mathcal{L}_{0}$, Lemma 5.3(1) implies that there is a pair of $(r-2, r+3)$ edges that also form an essential loop on $\widehat{T}$. It follows that we may choose an annulus $A$ in $\widehat{T}$ containing the edges of the configuration $C_{ \pm}(r)$, as in Lemma 5.2, so that the vertices $s, s+1, r-2, r+3$ lie outside $A$. But then $\left|A \cap K_{\gamma}\right| \leq t-4 \leq t / 2+1$, contradicting Lemma 5.2(2).

Case 2. $t=6$.

By Theorem 5.6, $\alpha_{x} \leq 3$ for all labels $x$, and hence $|\mathcal{L}|=6$ by Theorem 5.5. Therefore (taking $r=1$ without loss of generality) $G_{Q}$ contains a 12-Scharlemann cycle of length 2 , and $\mathcal{L}_{0} \subset\{5,6,3,4\}$.

If 4 or $5 \in \mathcal{L}_{0}$, then by Lemma 5.3(1) there is an essential loop of $(4,5)$ edges on $\widehat{T}$. We may therefore choose the annulus $A$ of Lemma 5.2 so that vertices 4 or 5 lie outside $A$. Hence $\left|A \cap K_{\gamma}\right|=4$, contradicting Lemma 5.2(2).

On the other hand, since $|\mathcal{S}| \leq 4$, we must have $\left|\mathcal{L}_{0}\right| \geq 2$. Therefore $\mathcal{L}_{0}=\{6,3\}$ and $\mathcal{S}=\{1,2,4,5\}$. But then $G_{Q}$ contains 12- and 45Scharlemann cycles of length 2 or 3 , contradicting Theorem 3.9.

\section{Klein bottle; the case $t \geq 4$.}

In this section and the next we will assume that $K(\gamma)$ contains an embedded Klein bottle $\widehat{S}$. Note that $\widehat{S}$ is incompressible. For let $c$ be a simple closed curve in $\widehat{S}$ bounding a disk in $K(\gamma)$. Then $c$ is orientation-preserving in $K(\gamma)$. If $c$ does not bound a disk on $\widehat{S}$ then the surgery of $\widehat{S}$ along $c$ would produce in $K(\gamma)$ either a non-separating 2-sphere or two disjoint projective planes, according as $c$ is non-separating or separating on $\widehat{S}$. But this contradicts the fact that $H_{1}(K(\gamma))$ is finite cyclic (since $\Delta>1$ ).

We suppose that $\widehat{S}$ is chosen to intersect $K_{\gamma}$ transversely and to minimize $\left|\widehat{S} \cap K_{\gamma}\right|$. Then $S=\widehat{S} \cap E(K)$ is incompressible in $E=E(K)$. The incompressibility of $S$ then implies the boundary incompressibility of $S$ (noting that a boundary compressing disk in the case $\left|\widehat{S} \cap K_{\gamma}\right|=1$ allows us to isotop $K_{\gamma}$ onto $\widehat{S}$ - contradicting the fact that $K$ is neither a satellite nor a torus knot). Let $\widehat{T}$ be the torus that is the boundary of a regular neighborhood of $\widehat{S}$, and let $S, T$ be the intersection of $\widehat{S}, \widehat{T}$ respectively with $E$. Let $t=|\partial T|=2|\partial S|$. Theorem 6.1 of this section and Theorem 7.1 of Section 7 imply Theorem 1.3.

In this section we prove 
Theorem 6.1. Suppose that $K(\gamma)$ contains a Klein bottle, with $t \geq 4$. Then $\Delta(\gamma, \mu)=1$.

Before proving Theorem 6.1, we give a general discussion of the context in which we will be working in this section and the next.

We begin with the following lemma.

Lemma 6.2. If $K(\gamma)$ contains a Klein bottle then $\Delta(\gamma, \mu)$ is odd.

Proof. Let $F$ be a closed non-orientable surface embedded in a closed orientable 3-manifold $M$. The homology exact sequence of the pair $(M, M-F)$ gives an exact sequence

$$
H_{1}(M) \rightarrow H_{1}(M, M-F) \rightarrow H_{0}(M-F) .
$$

Now $H_{0}(M-F)$ is free abelian, while, by Alexander duality, $H_{1}(M, M-F) \cong H^{2}(F) \cong \mathbb{Z}_{2}$. Hence $H_{1}\left(M ; \mathbb{Z}_{2}\right) \neq 0$. But if $\gamma$ is parametrized as $m / n$ in the usual way, then $H_{1}(K(\gamma)) \cong \mathbb{Z}_{|m|}$ and $\Delta(\gamma, \mu)=n$. Since $(m, n)=1$, the result follows.

Thus we assume in the next two sections that $\Delta(\gamma, \mu) \geq 3$ and arrive at a contradiction, when $t \geq 4$ in this section and when $t=2$ in Section 7 .

Let $p_{+}, p_{-}$be two points in $S^{3}$ and write $S^{3}-\left\{p_{+}, p_{-}\right\}=\widehat{Q} \times(-1,1)$ where $\widehat{Q}$ is a 2 -sphere. Let $S$ be as above. Then $S$ is a properly embedded, incompressible, boundary incompressible surface in $E$, whose boundary components are copies of the curve $\gamma$. Lemma 4.4 of [Ga, p.491] says that, after an isotopy of $K$, we can find a $\widehat{Q}=\widehat{Q} \times\{i\}$, for some $i$, such that:

(1) $\widehat{Q}$ intersects $K$ transversely. Thus $Q=\widehat{Q} \cap E$ is a properly embedded planar surface in $E$ such that each component of $\partial Q$ is a copy of the meridian $\mu$ of $K$.

(2) $Q$ intersects $S$ transversely and no arc component of $Q \cap S$ is parallel in $Q$ to $\partial Q$ or parallel in $S$ to $\partial S$.

By taking a small enough neighborhood, $X$, of $\widehat{S}$, we may assume that $Q \cap\left(X-\operatorname{nhd}\left(K_{\gamma}\right)\right)=(Q \cap S) \times[-\varepsilon, \varepsilon]($ note that these $I$-bundles over $Q \cap S$ are not twisted since they lie in $Q$ ). That is, $Q \cap T$ in $Q$ is the "double" of $Q \cap S$ in $Q$, and each component of $Q \cap S$ is double covered by two components of $Q \cap T$, as $T$ double covers $S$. Thus we may also conclude that 
(3) $Q$ intersects $T$ transversely and no arc component of $Q \cap T$ is parallel in $Q$ to $\partial Q$ or parallel in $T$ to $\partial T$.

We are now in the context of the first part of Section 2. As is done there, define the labelled graphs of intersection, $G_{Q}$ in $\widehat{Q}$ and $G_{T}$ in $\widehat{T}$. Also, define the labelled graphs $G_{Q}^{S}$ in $\widehat{Q}$ and $G_{S}$ in $\widehat{S}$ coming from the arc components of $Q \cap S$.

Let $c$ be a simple closed curve component of $Q \cap S$. By the incompressibility of $S, c$ must bound a disk on $S$. Thus we may get rid of $c$ by surgery. That is, we may assume that no simple closed curve of $Q \cap S$ bounds a disk in $Q$. But this implies that no simple closed curve of $Q \cap T$ bounds a disk in $Q$. Thus, as in Section 3 we will assume that no disk face of $G_{Q}^{S}, G_{Q}$ contains a simple closed curve component of $Q \cap S, Q \cap T$ (resp.).

Just as $\widehat{T}$ divides $K(\gamma)$ into a black side, $X$, and a white side, $X^{\prime}$, the faces of $G_{Q}$ are divided into black and white faces. Furthermore, each black face of $G_{Q}$ is a bigon and corresponds to an edge of $G_{Q}^{S}$.

The proofs in Section 2 go through for the pair $G_{Q}$ and $G_{T}$. In particular, Corollary 2.7 becomes

Theorem 6.3. $G_{Q}$ contains a great web.

However, the proofs in Section 3 usually rely on the incompressibility of $\widehat{T}$ and the fact that $\left|\widehat{T} \cap K_{\gamma}\right|$ is minimal. We no longer have the incompressibility of $\widehat{T}$ and the minimality is with respect to $\left|\widehat{S} \cap K_{\gamma}\right|$. We need to prove the analogs of some of these results under this assumption.

Theorem 6.4. If $t \geq 4$ then no Scharlemann cycle in $G_{Q}$ bounds a white face.

Proof. Let $\sigma$ be a Scharlemann cycle in $G_{Q}$ bounding a white face, $f$, of $G_{Q}$. Then this corresponds to a Scharlemann cycle, $\sigma^{\prime}$, in $G_{Q}^{S}$ bounding a white face $f^{\prime}$. Let $x, y$ be the labels of $\sigma^{\prime}$ (since $t \geq 4, x$ and $y$ will be distinct) and let $H$ be the annulus on $\partial E$ between the components $x$ and $y$ of $S$. Then tubing $S$ along $H$ and compressing along $f^{\prime}$ gives a new Klein bottle in $K(\gamma)$ that intersects $K_{\gamma}$ fewer times than $\widehat{S}$, contradicting the minimality of $\widehat{S}$.

Theorem 6.5. If $\sigma_{1}, \sigma_{2}$, and $\sigma_{3}$ are Scharlemann cycles in $G_{Q}$, then two of them must have the same pair of labels. 


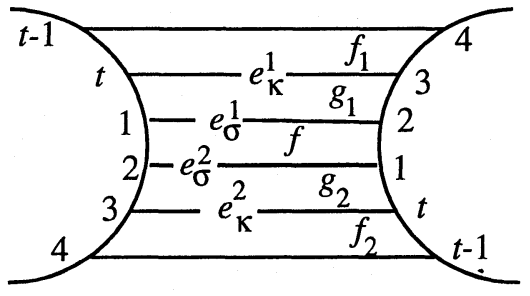

$G_{Q}$

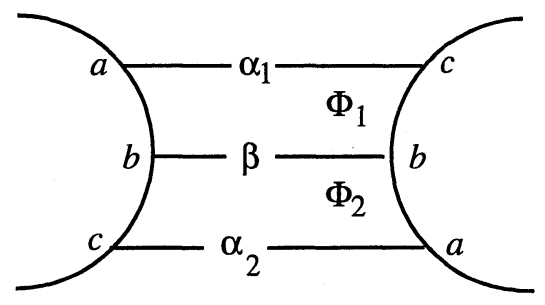

$G_{Q}^{S}$

Figure 6.1.

Proof. Let $f_{1}, f_{2}, f_{3}$ be the faces of $G_{Q}$ bounded by $\sigma_{1}, \sigma_{2}, \sigma_{3}$ (resp.). By Theorem 6.4, these must all be black faces of $G_{Q}$ and hence bigons. Assume for contradiction that the pairs of labels of the Scharlemann cycles are different. Since $f_{1}, f_{2}, f_{3}$ are all black, these label pairs must be disjoint. Let $\left\{k_{i}, k_{i}+1\right\}$ be the labels of $\sigma_{i}$. Let $H_{i}$ be that part of $V_{\gamma}$ running from component $k_{i}$ to component $k_{i}+1$ of $\partial T$. Let $F_{i}$ be the Möbius band obtained by taking $H_{i} \cup f_{i}$ and radially shrinking $H_{i}$ to its core. Then $F_{i}, i=1,2,3$, are disjoint Möbius bands properly embedded in $X$. But this is impossible.

Theorem 6.6. If $t \geq 6$ then $G_{Q}$ does not contain an extended Scharlemann cycle.

Proof. Assume for contradiction that $\kappa$ is an extended Scharlemann cycle in $G_{Q}$. Let $\sigma$ be the Scharlemann cycle interior to $\kappa$. Without loss of generality we may assume that the labels of the edges of $\sigma$ are $\{1,2\}$ and, consequently, the labels of the edges of $\kappa$ are $\{t, 3\}$. Let $f$ be the face of $G_{Q}$ bounded by $\sigma$. By Theorem 6.4, $f$ must be a black face and hence a bigon. Let $e_{\sigma}^{1}, e_{\sigma}^{2}$ be the edges of $\sigma$ and $e_{\kappa}^{1}, e_{\kappa}^{2}$ be the parallel edges of $\kappa$. For $i=1,2$, let $g_{i}$ be the bigon (white) face of $G_{Q}$ representing the parallelism of $e_{\kappa}^{i}$ and $e_{\sigma}^{i}$. Let $f_{1}, f_{2}$ be the black faces contiguous to $e_{\kappa}^{1}, e_{\kappa}^{2}$. See Figure 6.1.

In $G_{Q}^{S}, f, f_{1}, f_{2}$ go to edges $\beta, \alpha_{1}, \alpha_{2}$ and $g_{1}, g_{2}$ go to faces $\Phi_{1}, \Phi_{2}$. Note that the endpoints of the edge $\beta$ in $G_{Q}^{S}$ have the same label, say $b$, and the edges $\alpha_{1}$ and $\alpha_{2}$ have the same pair of distinct labels, say $\{a, c\}$. See Figure 6.1 .

Let $H_{a, c}$ be the part of $V_{\gamma}$ running between components $a$ and $c$ of $\partial S$. Let $A$ be the Möbius band obtained by taking $H_{a, c} \cup \Phi_{1} \cup \Phi_{2}$ and shrinking 
$H_{a, c}$ radially to its core. Let $\hat{\alpha}, \hat{\beta}$ be the simple closed curves in $\widehat{S}$ obtained from $\alpha_{1} \cup \alpha_{2}, \beta$ by shrinking the vertices of $G_{S}$ to points. Then $A$ intersects $\widehat{S}$ in $\partial A=\hat{\alpha}$ and in its centerline $\hat{\beta}$. The face $f$ of $G_{Q}$ gives rise to a twisted $I$-bundle over $\hat{\beta}$ that is normal to $\widehat{S}$, thus $\hat{\beta}$ must be orientation-reversing in $\widehat{S}$ (since $K(\gamma)$ is orientable). On the other hand, the faces $f_{1}$ and $f_{2}$ together give rise to a trivial $I$-bundle over $\hat{\alpha}$ that is normal to $\widehat{S}$, thus $\hat{\alpha}$ is orientation-preserving in $\widehat{S}$. Note that $\hat{\alpha}$ cannot bound a disk in $\widehat{S}$ since this disk along with $A$ would be a projective plane in $K(\gamma)$ contradicting [GLu3] (in fact one could avoid this reference to [GLu3] and the need that $\Delta(\gamma, \mu)>1$, by following the argument here and taking $\widehat{S}$ to be the surface intersecting $K_{\gamma}$ minimally among all projective planes and Klein bottles). Since $\hat{\alpha}, \hat{\beta}$ are disjoint, embedded loops in $\widehat{S}, \hat{\alpha}=\partial A$ must bound a Möbius band $F$ in $\widehat{S}$ disjoint from $\hat{\beta}$. Let $\widehat{P}$ be the Klein bottle $A \cup F$. We may now perturb $\widehat{P}$ to be transverse to $K_{\gamma}$ and have fewer intersections with $K_{\gamma}$, thereby contradicting the minimality of $\widehat{S}$.

We now observe that the conclusion of Theorem 4.3 holds in our present setting. Let $\mathcal{L}$ be the set of labels of $G_{Q}$ that are labels of Scharlemann cycles of length 2 in $G_{Q}$.

Theorem 6.7. If $t \geq 6$ then $|\mathcal{L}| \geq 4 t / 5$.

Proof. The proof is exactly the same as that of Theorem 4.3, using Theorem 6.3 instead of Corollary 2.7, and Theorem 6.6 instead of Theorem 3.2.

We can now prove Theorem 6.1 when $t \geq 6$.

Proof of Theorem 6.1 when $t \geq 6$. By Theorem 6.7 there must be at least five labels that appear as labels of Scharlemann cycles of length 2 in $G_{Q}$. But this contradicts Theorem 6.5.

The rest of this section will be devoted to proving Theorem 6.1 in the case $t=4$.

So suppose $t=4$, and consider a black face of $G_{Q}$ whose vertices are parallel. Such a face is a bigon, and is either a face bounded by a 12Scharlemann cycle, or a 34-Scharlemann cycle, or has label pair $\{1,2\}$ at one vertex and $\{3,4\}$ at the other. We will call these a 12-bigon, 34-bigon, and mixed bigon, respectively. 


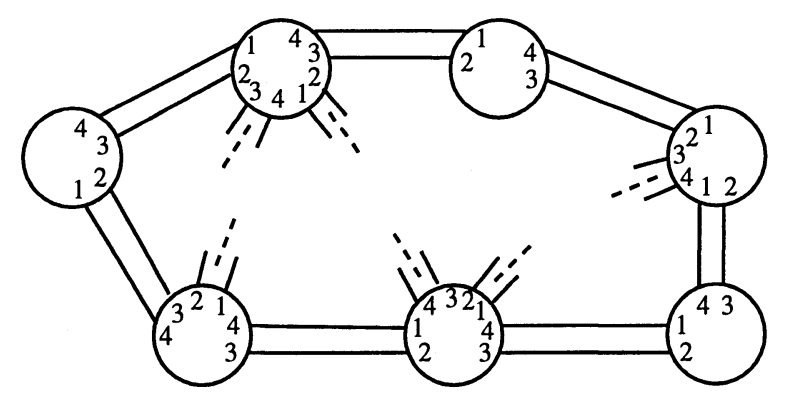

Figure 6.2.

Lemma 6.8. Suppose $t=4$. Then any disk bounded by a cycle of mixed bigons in $G_{Q}$ must contain vertices in its interior.

Proof. Consider an innermost cycle that contains no vertices in its interior. If the disk it bounds contains no black faces in its interior then it forms a Scharlemann cycle bounding a white face of $G_{Q}$, contradicting Theorem 6.4. Thus it must contain black bigons in its interior. But an outermost black bigon would have to be a mixed bigon (see Figure 6.2), contradicting our assumption that the cycle is innermost.

Let $\Lambda$ be the great web guaranteed by Theorem 6.3. Every black face of $G_{Q}$ is a bigon and we may assume an edge of $G_{Q}$ is in $\Lambda$ if and only if the corresponding black bigon is in $\Lambda$. Thus the definition of a great web says that all but at most two of the black bigons incident to vertices of $\Lambda$ are in $\Lambda$. From this one calculates that $\Lambda$ contains at least $3 V-1$ black bigons, where $V$ is the number of vertices of $\Lambda$. Because the vertices of $\Lambda$ are parallel, any black bigon in $\Lambda$ is either a 12-bigon, a 34-bigon, or a mixed bigon.

Lemma 6.9. Suppose $t=4$. Then $G_{Q}$ contains a 12-bigon and a 34-bigon .

Proof. Suppose for contradiction that $G_{Q}$ contains no 12-bigon, say. Then every bigon in $\Lambda$ with label-pair $\{1,2\}$ at one end is a mixed bigon. Hence the number of mixed bigons is at least $3 V-2 \geq V$. But this would give a cycle of mixed bigons of the kind prohibited by Lemma 6.8 .

Let $\sigma$ be a 12-bigon and $\tau$ a 34-bigon. By the proof of Lemma 3.1 (using the Klein bottle and its irreducible neighborhood in place of the 
incompressible torus) the edges of $\sigma$ and the edges of $\tau$ form disjoint essential simple closed curves on $\widehat{T}$. From this one sees that if $\rho$ is any other 12-bigon in $\Lambda$, then one edge of $\rho$ will be parallel on $G_{T}$ to one edge of $\sigma$ and the other edge of $\rho$ parallel to the other edge of $\sigma$. Similarly, the edges of any 34-bigon will be parallel in $G_{T}$ to the edges of $\tau$.

Lemma 6.10. Suppose $t=4$. Then there must be vertices of $G_{Q}$ on either side of a cycle of 12-bigons (or 34-bigons).

Proof. Suppose $G_{Q}$ contains a cycle of, say, 12-bigons one side of which contains no vertices of $G_{Q}$. Picking one edge from each of these bigons (see the comment preceding this lemma) we obtain a collection of edges $\left\{e_{1}, e_{2}, \ldots, e_{m}\right\}$ which in $G_{Q}$ bound a disk $D$ with no vertices in its interior and which in $G_{T}$ are parallel edges connecting vertices 1 and 2 . The argument from [GLi, p.130, Case (2)] shows that nhd( $\partial E \cup D \cup \bigcup\{$ bigon faces of $G_{T}$ between the edges $\left.e_{i}\right\}$ ) forms a cable space. (Note that if two edges $e_{i}, e_{j}$ had the same label ( 1 or 2$)$ at the same vertex of the cycle, then there would be more than $q$ parallel edges of $G_{T}$ and we could apply the full argument of [GLi, Section 5] to obtain a different cabling.) But our hypothesis on $K$ implies that $E$ does not contain a cable space.

Proof of Theorem 6.1 when $t=4$. Let $\Lambda$ be the great web described above. Since $\Lambda$ contains at least $3 V-1$ black bigons, there must be at least $V$ black bigons in $\Lambda$ all of which are either 12-bigons, or 34-bigons, or mixed bigons. But this would give a cycle of such bigons bounding a disk with no vertices in its interior, contradicting Lemmas 6.8 and 6.10 .

\section{Klein bottle; the case $t=2$.}

In the context of the first part of Section 6 we prove

Theorem 7.1. Suppose that $K(\gamma)$ contains a Klein bottle, with $t=2$. Then $\Delta(\gamma, \mu)=1$.

So let $\widehat{S}$ be an embedded Klein bottle in $K(\gamma)$ that intersects $K_{\gamma}$ once, and let $\widehat{T}, G_{T}, G_{Q}, G_{Q}^{S}$ be as in the first part of Section 6 through Theorem 6.3. We assume for contradiction that $\Delta(\gamma, \mu)>1$. By Lemma 6.2 


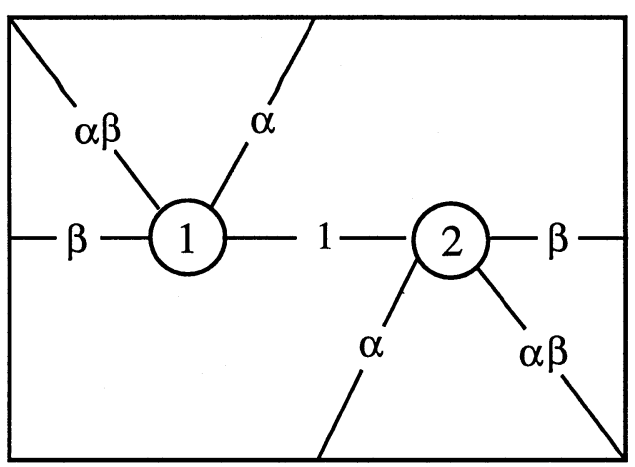

Figure 7.1.

$\Delta(\gamma, \mu)$ must be odd. We assume that $\Delta(\gamma, \mu)=3$, however the same argument works for $\Delta(\gamma, \mu) \geq 5$.

Theorem 6.3 now guarantees the existence of a great web, $\Lambda$, in $G_{Q}$. Thus $\Lambda$ contains at most two ghost edges. If $\Lambda$ contains any ghost edges we may assume there are exactly two ghost edges which cobound a black bigon. A black bigon of $G_{Q}$ connecting parallel vertices corresponds to an orientation-reversing curve on $\widehat{S}$ (the bigon is a Scharlemann cycle that gives rise to a Möbius band normal to $\widehat{S}$ of which this curve is a core). Since any two such curves intersect in a single point on $\widehat{S}$, there are at most two isotopy classes of such curves. Thus there are at most two isotopy classes of such edges in $G_{S}$. One such edge class in $G_{S}$ gives rise to two edge classes in $G_{T}, 1$ and $\beta$, which are the isotopy classes of edges of the corresponding black bigon in $G_{T}$. The other edge class in $G_{S}$ yields edge classes $\alpha$ and $\alpha \beta$ on $G_{T}$. After a homeomorphism of $\widehat{T}$ we assume these edge classes of $G_{T}$ appear as in Figure 7.1. For now consider $\Lambda$ as a graph in the 2-sphere, by adding an outside face to $\Lambda$. If $\Lambda$ has ghost edges then they are incident to a single vertex of $\Lambda$ which we call the exceptional vertex of $\Lambda$. The black faces of $\Lambda$ are bigons with edges in classes 1 and $\beta$ and in classes $\alpha$ and $\alpha \beta$.

Let $\Gamma$ be an oriented dual graph defined as follows: the vertices of $\Gamma$ are the white faces of $\Lambda$, the faces of $\Gamma$ are the vertices of $\Lambda$, and an edge $e$ of $\Gamma$ is an edge dual to a black face $f$ of $\Lambda$ oriented so that the edge of $f$ in class 1 or $\alpha$ is at the tail of $e$ and the edge of $f$ in class $\beta$ or $\alpha \beta$ is at the head of e. See Figure 7.2.

Claim 7.2. $\Gamma$ contains a sink or source which is not dual to the outside face. 


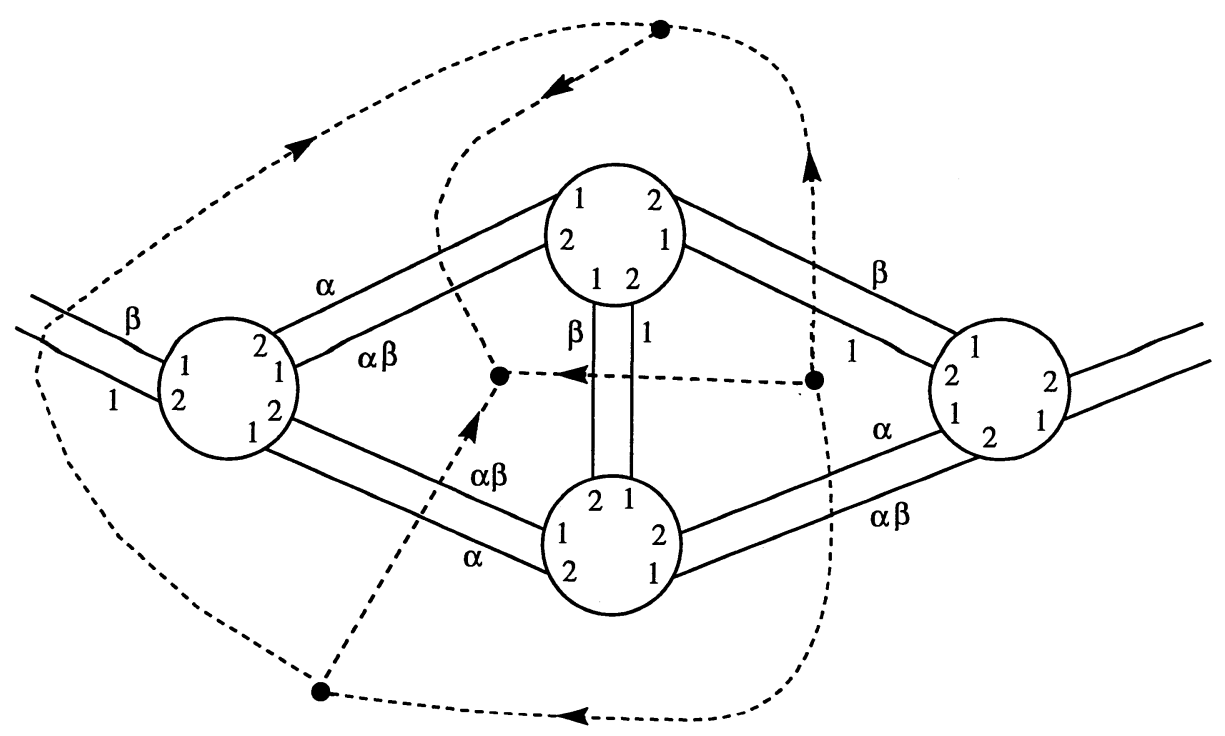

Figure 7.2.

A vertex of $\Lambda$ which is not the exceptional vertex cannot correspond to an oriented cycle in $\Gamma$ : otherwise that vertex, $V$, would have two edges in the same edge class (e.g., $\beta$ ) incident to it on the same label. See Figure 7.3. The argument of [GLi, Section 5] shows that $E(K)$ would then contain a cable space contradicting our assumption on $K$.

Thus if there is an oriented cycle face in $\Gamma$, it is dual to the exceptional vertex of $\Lambda$. In this case, the outside face of $\Lambda$ is not dual to a sink or source of $\Gamma$. Since $\Gamma$ contains at least two elements in \{sinks, sources, cycle faces\}, the claim now follows.

Claim 7.3. $G_{Q}$ contains a white face all of whose edges are in classes 1 and $\alpha$.

Proof. A source of $\Gamma$ corresponds to a white face of $\Lambda$ whose edges are in classes 1 and $\alpha-$ a sink to a white face with edges in classes $\beta$ or $\alpha \beta$. Applying Claim 7.2 and possibly relabelling the edges of $G_{T}$ gives a white face of $G_{Q}$ with edges labelled 1 and $\alpha$.

Lemma 7.4. There is a Klein bottle in $K(\gamma)$ containing $K_{\gamma}$ as an orientation-reversing curve. 


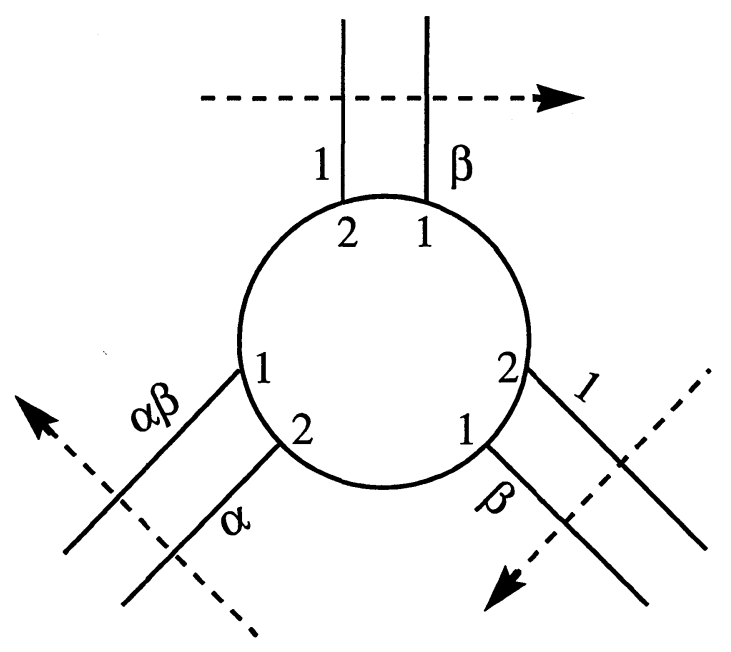

Figure 7.3.

Proof. By Claim 7.3, $G_{Q}$ contains a white face $f$ bounded by edges in classes 1 and $\alpha$. Consider the extended Scharlemann cycle $g$ obtained by adding contiguous black bigons to $f$. By the proof of Lemma 3.1, modified as noted in the proof of Lemma 6.9, $f$ must contain edges in both classes 1 and $\alpha$; hence, $g$ must contain edges in both classes $\beta$ and $\alpha \beta$. Label the vertices of $g x_{1}, \ldots, x_{n}$ and note that the $x_{i}$ must be distinct since two of the white faces incident to the $x_{i}$ contain $\alpha \beta$ or $\beta$ edges. On each $x_{i}$ let $\lambda_{i}$ be the interval on $g \cap x_{i}$ between the two edges whose endpoints are labelled 1 at $x_{i}$. See Figure 7.4.

Look at these $\lambda_{i}$ in $K(\gamma)$, in particular in $T \cup \partial E$. Each $\lambda_{i}$ has one endpoint, $\partial^{1} \lambda_{i}$, in an edge of class 1 or $\alpha$ and an endpoint, $\partial^{2} \lambda_{i}$, in an edge of class $\beta$ or $\alpha \beta$. On vertex 1 of $G_{T}$ there are disjoint intervals, $I_{1}$ and $I_{2}$, such that $\left\{\partial^{1} \lambda_{i}\right\}_{i=1, \ldots, n} \subset I_{1},\left\{\partial^{2} \lambda_{i}\right\}_{i=1, \ldots, n} \subset I_{2}$. Choose $I_{1}, I_{2}$ to be the shortest such, then $\partial I_{k} \subset\left\{\partial^{k} \lambda_{i}\right\}_{i=1, \ldots, n}$. Label $x_{1}, \ldots, x_{n}$ so that $\partial I_{1}=\left\{\partial^{1} \lambda_{1}, \partial^{1} \lambda_{n}\right\}$. In particular, take $\partial^{1} \lambda_{1}$ to be incident to an edge in class 1 and $\partial^{1} \lambda_{n}$ to be incident to an edge of class $\alpha$. See Figure 7.5.

Claim 7.5. $\partial^{2} \lambda_{1}$ is contained in an edge of class $\alpha \beta$, and $\partial^{2} \lambda_{n}$ is contained in an edge of class $\beta$. 


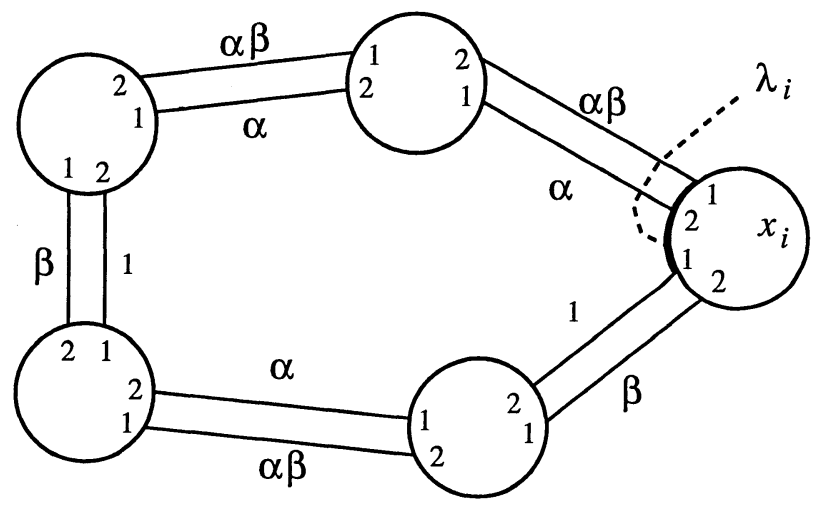

Figure 7.4.

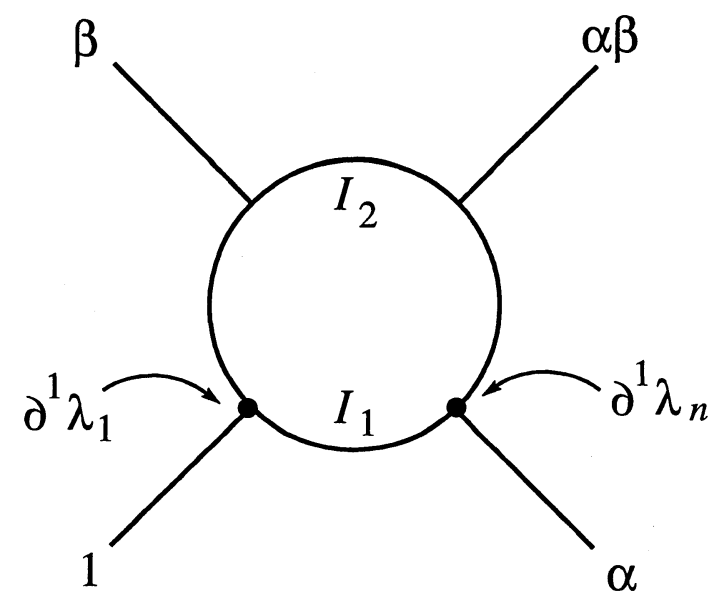

Figure 7.5. 


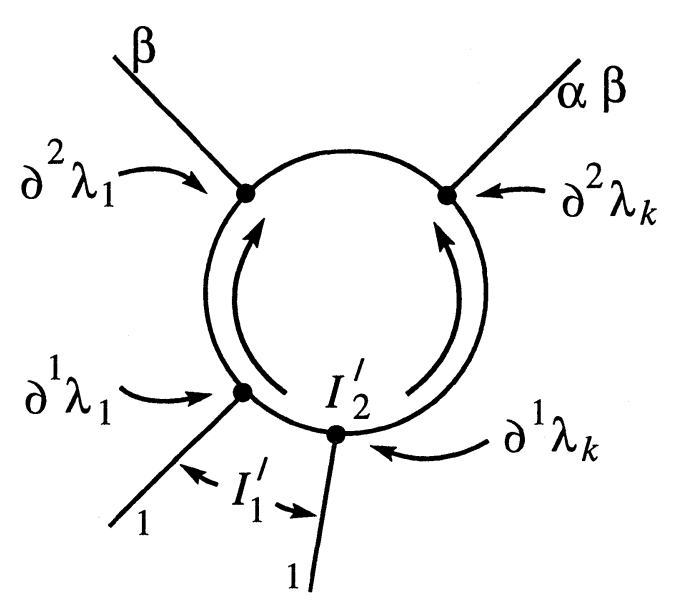

Figure 7.6.

Proof. Assume for contradiction that $\partial^{2} \lambda_{1}$ is in an edge of class $\beta$. Then there is a $\lambda_{k}$ such that $\partial^{1} \lambda_{k}$ is in an edge of class 1 and $\partial^{2} \lambda_{k}$ is in an edge of class $\alpha \beta$ (because there is corner of $f$ that runs from label 1 on an edge in class 1 to label 2 on an edge in class $\alpha$ ). Let $I_{1}^{\prime}$ be the subinterval of $I_{1}$ between $\partial^{1} \lambda_{1}$ and $\partial^{1} \lambda_{k}$. Then $\lambda_{1} \cup I_{1}^{\prime} \cup \lambda_{k}$ is isotopic in $\partial E$ (rel its endpoints) to an arc $I_{2}^{\prime}$ in vertex 1 that will contain $I_{1}^{\prime}$ in its interior. See Figure 7.6. But this is impossible as the number of labels in $I_{1}^{\prime}$ would have to be the same as the number in $I_{2}^{\prime}$ (these labels are paired by $\operatorname{arcs}$ in $\partial Q$ ).

This proves that $\partial^{2} \lambda_{1}$ is in an edge of class $\alpha \beta$. Similarly $\partial^{2} \lambda_{n}$ is in an edge of class $\beta$.

Claim 7.5 shows that the $\operatorname{arc} \lambda_{1} \cup I_{1} \cup \lambda_{n}$ is isotopic on $\partial E$ (rel endpoints) to $I_{2}$. In particular $\lambda_{1} \cup I_{1} \cup \lambda_{n} \cup I_{2}$ bounds an embedded disk $D$ in $\partial E$.

Let $p: \widehat{T} \rightarrow \widehat{S}$ be the 2 -fold covering projection defined by the twisted $I$ bundle structure of the regular neighborhood of $\widehat{S}$ with boundary $\widehat{T}$. Then $I_{1}^{\prime}=p\left(I_{1}\right), I_{2}^{\prime}=p\left(I_{2}\right)$ are disjoint arcs in the single component of $\partial S$, and the disk $D$ determines an embedded disk $D^{\prime}$ in $\partial E$ which meets $\partial S$ in $I_{1}^{\prime} \cup I_{2}^{\prime}$ (see Figure 7.7). Let $f^{\prime}$ be the face of $G_{Q}^{S}$ corresponding to the face $f$ of $G_{Q}$. Note that the corners of $f^{\prime}$ lie in $D^{\prime}$. Thickening $D^{\prime}$ gives a 1-handle $H \subset K(\gamma)$ whose attaching region is a pair of disjoint disks in $\widehat{S}$ containing $I_{1}^{\prime}$ and $I_{2}^{\prime}$. Surgering $\widehat{S}$ along $H$ gives a non-orientable surface $\widehat{F}=(\widehat{S} \cup \partial H)-(\widehat{S} \cap \partial H)$. We may isotop $f^{\prime}$ off Int $H$, so that $\partial f^{\prime}$ becomes a non-separating, orientation preserving curve on $\widehat{F}$. 


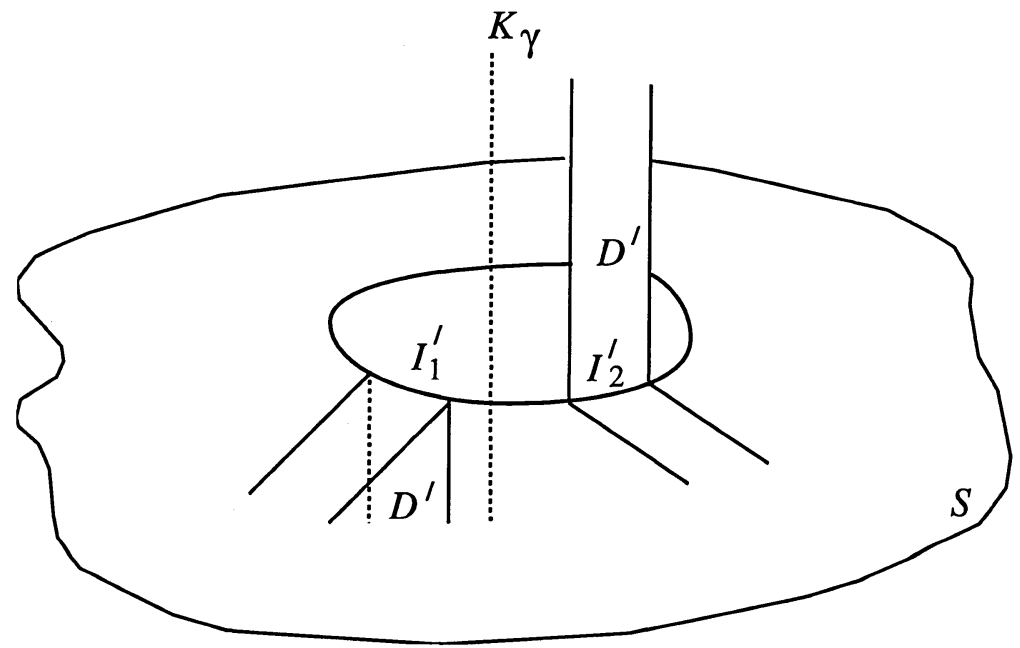

Figure 7.7 .

Surger $\widehat{F}$ along $f^{\prime}$, giving a new Klein bottle $\widetilde{S}$. Note that $\widetilde{S}$ intersects $K_{\gamma}$ in a single point, but as in Figure $7.8 K_{\gamma}$ can now be isotoped to lie on $\widetilde{S}$ (as the union of two arcs - one on $\partial H$ and the other in the disk $\widehat{S}-\operatorname{Int} S$ ). Finally, note that $K_{\gamma}$ is 1-sided in $\widetilde{S}$.

$\square$ (Lemma 7.4)

Proof of Theorem 7.1. By Lemma 7.4, $K_{\gamma}$ is contained as an orientationreversing curve in an embedded Klein bottle $\widetilde{S}$ in $K(\gamma)$. Then $\widetilde{S}-\operatorname{Int} V_{\gamma}$ is a Möbius band properly embedded in $E$. This means that $K$ is a $(2, \mathrm{n})$-cable knot, contradicting our assumption that $K$ is not a satellite or torus knot.

\section{Strong invertibility.}

Recall that a knot $K$ in $S^{3}$ is strongly invertible if there exists an orientation-preserving involution $h: S^{3} \rightarrow S^{3}$, with fixed-point set an unknotted circle $K_{0}$, such that $h(K)=K$ and $h \mid K$ is orientation-reversing. Note that $K_{0}$ meets $K$ in two points.

In this section we shall prove the following theorem.

Theorem 8.1. Suppose that $K(\gamma)$ contains an incompressible torus $\widehat{T}$, where $\Delta(\gamma, \mu) \geq 2$ and $t=\left|K_{\gamma} \cap \widehat{T}\right|=2$. Then $T=\widehat{T} \cap E(K)$ separates 


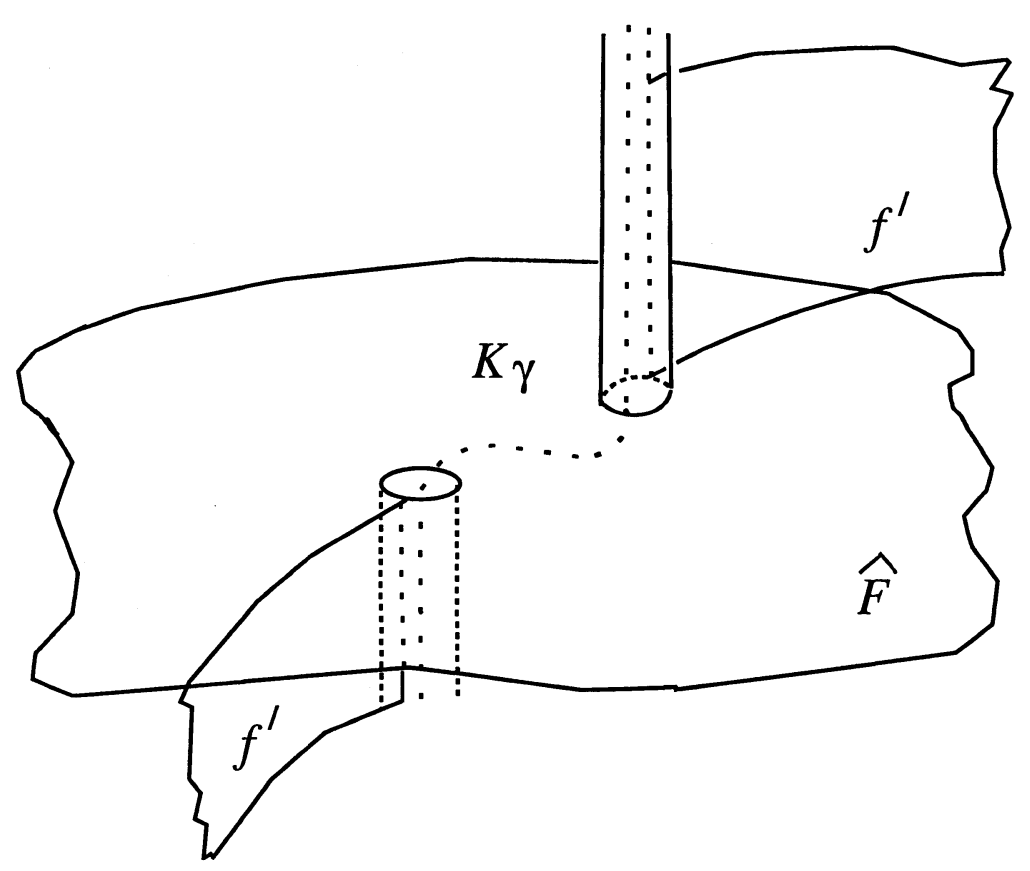

Figure 7.8.

$E(K)$ into two genus 2 handlebodies. In particular, $K$ is strongly invertible. Furthermore, the tunnel number of $K$ is at most 2.

Let $\widehat{T}$ and $T$ be as hypothesized in Theorem 8.1. Let $Q$ be a planar surface as described in Section 2 and $G_{Q}, G_{T}$ be the resulting graphs of intersection. $T$ separates $E$ into black and white sides $W$ and $W^{\prime}$, say, and a face of $G_{Q}$ is black or white according as it lies in $W$ or $W^{\prime}$.

Lemma 8.2. $G_{Q}$ contains a Scharlemann cycle of length 2 or 3 . Also, $G_{Q}$ contains a black Scharlemann cycle and a white Scharlemann cycle.

Proof. Let $\Lambda$ be the great web in $G_{Q}$ guaranteed by Corollary 2.7. Let $D$ be the disk bounded by $\Lambda$. Note that $\Lambda$ has at most two ghost labels. We consider $\Lambda$ as a graph in the disk $D$ (the outside region containing $\partial D$ will not be a face of this graph). Because the vertices of $\Lambda$ are parallel, any face of $\Lambda$ is a Scharlemann cycle of $G_{Q}$. We first show that $\Lambda$ contains a face of length at most three, thereby proving the first part of Lemma 8.2 We then show $\Lambda$ must contain both a black and white face, proving the second part. So assume for contradiction that each face of $\Lambda$ has length at least four. If 


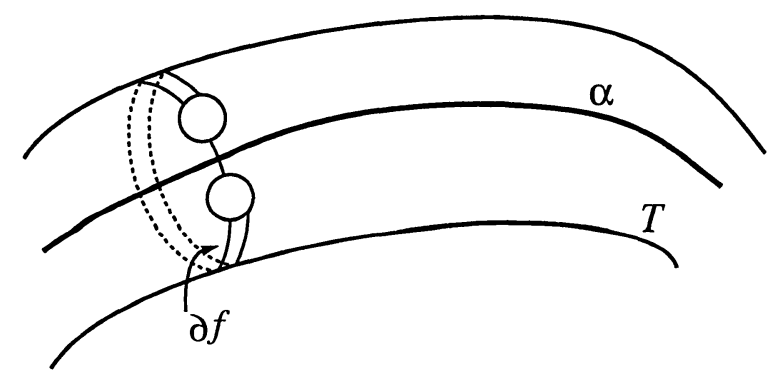

Figure 8.1.

$V, E, F$ are the vertices, edges, and faces (resp.) of $\Lambda$, then $4 F<2 E$. On the other hand, every vertex of $\Lambda$ without a ghost label has valence at least four. Since there are at most two ghost labels, $E \geq \frac{4 V-2}{2}=2 V-1$. Thus $1=V-E+F<\frac{E+1}{2}-E+\frac{E}{2}=\frac{1}{2}$, a contradiction.

Now assume that $\Lambda$ does not have a, say, white face. Then we may connect each white corner of a vertex of $\Lambda$ to $\partial D$ by an embedded arc, where these arcs are disjoint from the edges of $\Lambda$ and from each other. Let $\alpha_{1}$ and $\alpha_{2}$ be an outermost pair of these arcs, and let $v$ be the vertex at which they are incident. Then the two labels at $v$ between $\alpha_{1}$ and $\alpha_{2}$ in this outermost sector must be ghost labels for $\Lambda$. Since there are at least two such outermost sectors, $\Lambda$ must then have more than two ghost labels, a contradiction.

Lemma 8.3. $W$ and $W^{\prime}$ are handlebodies of genus 2.

Proof. Note that $\partial W$ and $\partial W^{\prime}$ are surfaces of genus 2. The faces bounded by the black and white Scharlemann cycles of Lemma 8.2 give compressions of $\partial W$ and $\partial W^{\prime}$ in $W$ and $W^{\prime}$ respectively. Since $E$ contains no essential torus, and since $T$ is incompressible in $E, W$ and $W^{\prime}$ contain no incompressible tori. Hence $W$ and $W^{\prime}$ are genus 2 handlebodies.

Lemma 8.4. $K$ has tunnel number at most 2 .

Proof. Let $f$ be the face in $G_{Q}$ bounded by a Scharlemann cycle of length at most 3 (guaranteed by Lemma 8.2) and assume $f$ is in $W$. Let $\alpha$ be a curve on $T$ which intersects $\partial f$ transversely once. See Figure 8.1. 


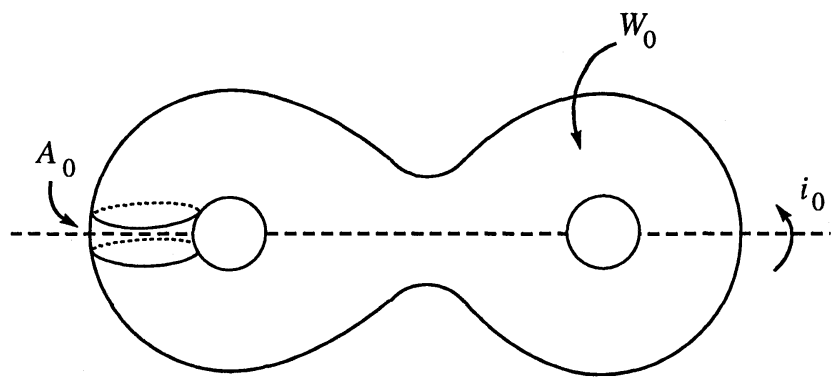

Figure 8.2.

Let $\beta_{1}, \beta_{2}$ be arcs in $T$ connecting different components of $\partial T$ such that $T-\operatorname{nhd}_{T}\left(\beta_{1} \cup \beta_{2}\right)$ is an annulus, $A$, whose core is $\alpha$. Since $\alpha$ has geometric intersection number one with the boundary of a disk in $W$, it is easy to see that $E(K)-\operatorname{nhd}_{E(K)}\left(\beta_{1} \cup \beta_{2}\right) \cong W \cup_{A} W^{\prime}$ is a handlebody. Thus $K$ has tunnel number at most 2 .

Lemma 8.5. One side of the incompressible torus in $K(\gamma)$ is a Seifert fiber space over a disk with two exceptional fibers. Furthermore, at least one of the exceptional fibers has order 2 or 3.

Proof. This follows from Lemma 8.2 and Theorem 3.8.

Proof of Theorem 8.1. We have shown that $E(K)$ is the union of two. genus 2 handlebodies along $T$. We need to show that this means $K$ is strongly invertible. Let $T, T^{\prime}$ be the copies of $T$ in $\partial W, \partial W^{\prime}$ respectively, and let $A, A^{\prime}$ be the annuli such that $\partial W=T \cup A, \partial W^{\prime}=T^{\prime} \cup A^{\prime}$. Thus $E=W \cup W^{\prime}$, glued by some homeomorphism $\varphi: T \rightarrow T^{\prime}$, and $\partial E=A \cup A^{\prime}$.

Let $W_{0}$ be a "model" handlebody of genus 2 , standardly embedded in $R^{3}$. Let $i_{0}: W_{0} \rightarrow W_{0}$ be the involution defined by rotating $W_{0}$ through $\pi$ about the axis indicated in Figure 8.2.

Let $h: W \rightarrow W_{0}, h^{\prime}: W^{\prime} \rightarrow W_{0}$ be homeomorphisms, and define involutions $i, i^{\prime}$ of $W, W^{\prime}$ respectively by $i=h^{-1} i_{0} h, i^{\prime}=h^{\prime-1} i_{0} h^{\prime}$. Let $A_{0}$ be the annulus in $\partial W_{0}$ shown in Figure 8.2. Note that $i_{0}\left(A_{0}\right)=A_{0}$, and that with respect to a suitable parametrization of $A_{0}$ as $S^{1} \times[-1,1], i_{0} \mid A_{0}$ is given by $(\theta, r) \mapsto(-\theta,-r)$.

Let $f: \partial W_{0} \rightarrow \partial W_{0}$ be an orientation-preserving homeomorphism such that $f(h(A))=A_{0}$. Then, as in [V], $f$ is isotopic to $g$ such that $i_{0} g=g i_{0}$. 
Composing $h$ with a homeomorphism of $W_{0}$ whose restriction to $\partial W_{0}$ is $g^{-1} f$ (and still calling the result $h$ ), we may suppose that $g h(A)=A_{0}$.

Similarly, we have a homeomorphism $g^{\prime}: \partial W_{0} \rightarrow \partial W_{0}$ such that $i_{0} g^{\prime}=$ $g^{\prime} i_{0}$ and $g^{\prime} h^{\prime}\left(A^{\prime}\right)=A_{0}$.

We wish to define an involution $j: E \rightarrow E$ by setting $j \mid W=i$, $j \mid W^{\prime}=i^{\prime}$. The condition that $j$ be well-defined is that $\varphi i=i^{\prime} \varphi: T \rightarrow T^{\prime}$. Since $i_{0}(g h)=(g h) i: T \rightarrow T_{0}$, and similar $i_{0}\left(g^{\prime} h^{\prime}\right)=\left(g^{\prime} h^{\prime}\right) i^{\prime}: T^{\prime} \rightarrow T_{0}$, this compatibility condition is equivalent to $\varphi_{0} i_{0}=i_{0} \varphi_{0}: T_{0} \rightarrow T_{0}$, where $\varphi_{0}=\left(g^{\prime} h^{\prime}\right) \varphi(g h)^{-1}: T_{0} \rightarrow T_{0}$. But it follows from [Bi, Theorem 3] that $\varphi_{0}$ is isotopic to $\psi_{0}: T_{0} \rightarrow T_{0}$ such that $\psi_{0} i_{0}=i_{0} \psi_{0}$. Then $\varphi$ is isotopic to $\psi=\left(g^{\prime} h^{\prime}\right)^{-1} \psi_{0}(g h): T \rightarrow T^{\prime}$ such that $\psi i=i^{\prime} \psi$. Thus we may define the involution $j: E \rightarrow E$ as desired.

Note that, parametrizing $\partial E=A \cup A^{\prime}$ as $S^{1} \times S^{1}$ in the obvious way, $j \mid \partial E$ is given by $(\theta, \varphi) \mapsto(-\theta,-\varphi)$. Now $S^{3} \cong E \cup S^{1} \times D^{2}$, where $S^{1} \times D^{2} \cong N(K)$, and where we may assume that the gluing homeomorphism $\partial E \rightarrow \partial\left(S^{1} \times D^{2}\right)$ is given by an element of $G L_{2}(\mathbb{Z})$. Hence $j$ extends to an involution of $S^{3}$ by setting $j(\theta,(r, \varphi))=(-\theta,(r,-\varphi)),(\theta,(r, \varphi)) \in S^{1} \times D^{2}$. This involution is the desired strong inversion of $K$.

Proof of Theorem 1.1. Suppose that $K(\gamma)$ contains an incompressible torus $\widehat{T}$, chosen to minimize $t=\left|\widehat{T} \cap K_{\gamma}\right|$, and suppose that $\Delta(\gamma, \mu) \geq 3$. By Theorem 4.1, we then have $t=2$. Hence, by Theorem $8.1, K$ is strongly invertible. But by [EM1], this implies that $\Delta(\gamma, \mu) \leq 2$, a contradiction.

In [EM2], Eudave-Muñoz constructs an infinite family of strongly invertible, hyperbolic knots in the 3-sphere, $k(\ell, m, n, p)$, such that $k(\ell, m, n, p)(\gamma)$ contains an essential torus for some $\gamma$ with $\Delta(\gamma, \mu)=2$. In fact, EudaveMuñoz shows that each side of the essential torus in $k(\ell, m, n, p)(\gamma)$ is a Seifert fiber space over a disk with two exceptional fibers.

Let $K$ be a hyperbolic knot in $S^{3}$ such that $K(\gamma)$ contains an essential torus where $\Delta(\gamma, \mu)=2$. Theorem 1.1 says that such a knot is extremal among those hyperbolic knots admitting Dehn surgeries containing essential tori. Theorem 1.2 says that $K$ is special. We end with a few questions asking exactly how special $K$ is.

Question 1. Does $K=k(\ell, m, n, p)$ for some values of $\ell, m, n$, and $p$ ?

Question 2. Is each side of the incompressible torus in $K(\gamma)$ Seifert fibered over the disk with two exceptional fibers? 
Towards Question 2, recall Lemma 8.5 and the fact that the answer is yes for the knots $k(\ell, m, n, p)$.

Question 3. Is $K$ a tunnel number one knot? In particular, does $k(\ell, m, n, p)$ have tunnel number one?

Added un proof. Eudave-Muñoz (private communication) has shown that $k(l, m, n, p)$ has tunnel number one.

Recall that Lemma 8.4 says that $K$ has tunnel number at most two.

\section{References.}

[Be] J. Berge, Some knots with surgeries yielding lens spaces, preprint.

[Bi] J. Birman, Mapping class groups and their relationship to braid groups, Communications on Pure and Applied Mathematics, 22 (1969), 213-238.

[BH] S.A. Bleiler, C.G. Hodgson, Spherical space forms and Dehn surgery, Topology, to appear.

[BZ1] S. Boyer, X. Zhang, Finite Dehn surgery on knots, preprint.

[BZ2] S. Boyer, X. Zhang, The semi-norm and Dehn filling, preprint.

[CGLS] M. Culler, C. McA. Gordon, J. Luecke, P.B. Shalen, Dehn surgery on knots, Ann. Math. 125(2) (1987), 237-300.

[EM1] M. Eudave-Muñoz, Essential tori obtained by surgery on a knot, PacificJ. Math., to appear.

[EM2] M. Eudave-Muñoz, Non-hyperbolic manifolds obtained by Dehn surgery on hyperbolic knots, Proceedings of the Georgia International Topology Conference (1993), to appear.

[Ga] D. Gabai, Foliations and the topology of 3-manifolds, III, J. Diff. Geom. 26 (1987), 479-536.

[GS] F. González-Acuña, H. Short, Knot surgery and primeness, Math. Proc. Camb. Phil. Soc. 99 (1986), 89-102.

[GLi] C. McA. Gordon, R.A. Litherland, Incompressible planar surfaces in 3manifolds, Topology and its Applications, 18 (1984), 121-144.

[GLu1] C. McA. Gordon, J. Luecke, Dehn surgeries on knots creating essential tori, II, preprint. 
[GLu2] C. McA. Gordon, J. Luecke, Knots are determined by their complements, J. Amer. Math. Soc. 2 (1989), 371-415.

[GLu3] C. McA. Gordon, J. Luecke, Only integral Dehn surgeries can yield reducible manifolds, Math. Proc. Camb. Phil. Soc. 102 (1987), 94-101.

[GLu4] C. McA. Gordon, J. Luecke, Reducible manifolds and Dehn surgery, Topology, to appear.

[HO] A. Hatcher, U. Oertel, Boundary slopes for Montesinos knots, Topology, 28 (1989), 453-480.

[Par] W. Parry, All types implies torsion, Proc. Amer. Math. Soc. 110 (1990), 871-875.

[Pat] R. Patton, Incompressible punctured tori in the complements of alternating knots, Math. Ann. 301 (1995), 1-22.

[R] D. Rolfsen, Knots and Links, Math. Lect. Ser. 7, Publish or Perish, Berkeley, California 1976.

[T] W. Thurston, Three dimensional manifolds, Kleinian groups, and hyperbolic geometry, Bull. Amer. Math. Soc. 6 (1982), 357-381.

[V] O. Ja. Viro, Linkings, 2-sheeted branched coverings, and braids, Mat. Sb. (N.S.), 87(129) (1972), 216-228, English translation: Math. USSR-Sb. 16 (1972), 223-236.

[Wu] Y.Q. Wu, The reducibility of surgered 3-manifolds, Topology and its Applications, 43 (1992), 213-218.

RECEIVEd JANUARY 31st, 1995.

DEPARTMENT OF MATHEMATICS

UNIVERSITY OF TEXAS

Austin, TX 78712 